\title{
Boundedness for Parametrized Littlewood-Paley Operators with Rough Kernels on Weighted Weak Hardy Spaces
}

\author{
Ximei Wei and Shuangping Tao \\ College of Mathematics and Statistics, Northwest Normal University, Lanzhou, Gansu 730070, China \\ Correspondence should be addressed to Shuangping Tao; taosp@nwnu.edu.cn
}

Received 27 April 2013; Accepted 18 June 2013

Academic Editor: Dashan Fan

Copyright (C) 2013 X. Wei and S. Tao. This is an open access article distributed under the Creative Commons Attribution License, which permits unrestricted use, distribution, and reproduction in any medium, provided the original work is properly cited.

The authors prove that the parametrized area integral $\mu_{\Omega, S}^{\rho}$ and $g_{\lambda}^{*}$ function $\mu_{\lambda}^{*, \rho}$ are bounded from the weighted weak Hardy space $W H_{w}^{1}\left(\mathbb{R}^{n}\right)$ to the weighted weak Lebesgue space $W L_{w}^{1}\left(\mathbb{R}^{n}\right)$ as $\Omega$ satisfies a class of the integral Dini condition, respectively.

\section{Introduction and Main Results}

Suppose that $\Omega \in L^{1}\left(S^{n-1}\right)$ is homogeneous of degree zero on $\mathbb{R}^{n}$ and satisfies

$$
\int_{S^{n-1}} \Omega\left(x^{\prime}\right) \mathrm{d} \sigma\left(x^{\prime}\right)=0,
$$

where $S^{n-1}$ denotes the unit sphere of $\mathbb{R}^{n}(n \geqslant 2)$ equipped with normalized Lebesgue measure $\mathrm{d} \sigma=\mathrm{d} \sigma\left(x^{\prime}\right), x^{\prime}=x /|x|$, $x \neq 0$. The parametrized area integral $\mu_{\Omega, S}^{\rho}$ and $g_{\lambda}^{*}$ function $\mu_{\lambda}^{*, \rho}$ are defined by

$$
\begin{aligned}
& \mu_{\Omega, S}^{\rho}(f)(x) \\
&=\left(\iint_{\Gamma(x)}\left|\frac{1}{t^{\rho}} \int_{|y-z|<t} \frac{\Omega(y-z)}{|y-z|^{n-\rho}} f(z) \mathrm{d} z\right|^{2} \frac{\mathrm{d} y \mathrm{~d} t}{t^{n+1}}\right)^{1 / 2}, \\
& \mu_{\lambda}^{*, \rho}(f)(x)=\left(\iint_{\mathbb{R}_{+}^{n+1}}\left(\frac{t}{t+|x-y|}\right)^{\lambda n}\right. \\
&\left.\times\left|\frac{1}{t^{\rho}} \int_{|y-z|<t} \frac{\Omega(y-z)}{|y-z|^{n-\rho}} f(z) \mathrm{d} z\right|^{2} \frac{\mathrm{d} y \mathrm{~d} t}{t^{n+1}}\right)^{1 / 2},
\end{aligned}
$$

respectively, where

$$
\begin{array}{r}
\Gamma(x)=\left\{(y, t) \in \mathbb{R}_{+}^{n+1}:|x-y|<t\right\}, \\
\mathbb{R}_{+}^{n+1}=\mathbb{R}^{n} \times(0, \infty), \quad \rho>0, \quad \lambda>1 .
\end{array}
$$

It is well known that Littlewood-Paley functions are very important tools in harmonic analysis and PDE (see [1-3]). Some well-known results related to the classical LittlewoodPaley operators can be seen in [4-8]. In 1999, inspired by Hörmander's work [9], when $\Omega$ satisfies the Lipschitz condition of $\alpha$, Sakamoto and Yabuta [10] established the $L^{p}(1<p<\infty)$ boundedness of the parametrized area integral $\mu_{\Omega, S}^{\rho}$ and the parametrized $g_{\lambda}^{*}$ function $\mu_{\lambda}^{*, \rho}$ and gave the boundedness on $B M O$ spaces and Campanato spaces. For any $0<\alpha \leqslant 1,1<q \leqslant \infty$, it is easy to see that the inclusion relationship

$$
\begin{aligned}
\operatorname{Lip}_{\alpha}\left(S^{n-1}\right) & \subsetneq L^{q}\left(S^{n-1}\right) \subsetneq L \log ^{+} L\left(S^{n-1}\right) \\
& \subsetneq H^{1}\left(S^{n-1}\right) \subsetneq L^{1}\left(S^{n-1}\right)
\end{aligned}
$$

holds. In 2002, Ding et al. [11] extended the previous $L^{p}$. boundedness to the case as $\Omega$ belongs to $L \log ^{+} L\left(S^{n-1}\right)$. In 2007, Ding et al. [12,13] gave the boundedness of the parametrized area integral $\mu_{\Omega, S}^{\rho}$ and $g_{\lambda}^{*}$ function $\mu_{\lambda}^{*, \rho}$ on the Hardy space and weak Hardy space when $\Omega$ satisfies a class of the integral Dini conditions. Recently, Wang and Liu [14] 
obtained the boundedness on the weighted Hardy space for the parametrized Littlewood-Paley operators with $\Omega$ satisfying the logarithmic type Lipschitz conditions. On the other hand, the boundedness properties of the intrinsic square functions on weighted weak Hardy spaces were studied by Wang in [15]. Inspired by the results mentioned previously, in this paper, we will study the boundedness of the parametrized area integral $\mu_{\Omega, S}^{\rho}$ and $g_{\lambda}^{*}$ function $\mu_{\lambda}^{*, \rho}$ on the weighted weak Hardy spaces.

Before stating our main results, let us recall some definitions. Firstly, let $\Omega\left(x^{\prime}\right) \in L^{q}\left(S^{n-1}\right), q \geqslant 1$. Then, the integral modulus $\omega_{q}(\delta)$ of continuity of order $q$ of $\Omega$ is defined by

$$
\omega_{q}(\delta)=\sup _{\|\gamma\| \leqslant \delta}\left(\int_{S^{n-1}}\left|\Omega\left(\gamma x^{\prime}\right)-\Omega\left(x^{\prime}\right)\right|^{q} \mathrm{~d} \sigma\left(x^{\prime}\right)\right)^{1 / q},
$$

where, $\gamma$ denotes a rotation on $S^{n-1}$ and $\|\gamma\|=\sup _{x^{\prime} \in S^{n-1}} \mid \gamma x^{\prime}-$ $x^{\prime} \mid$. The function $\Omega$ is said to satisfy the $L^{q}$-Dini condition, if

$$
\int_{0}^{1} \frac{\omega_{q}(\delta)}{\delta} \mathrm{d} \delta<\infty
$$

Secondly, given a weight function $w$ on $\mathbb{R}^{n}$, for $1 \leqslant p<\infty$, the weighted Lebesgue spaces is defined by

$$
L_{w}^{p}\left(\mathbb{R}^{n}\right)=\left\{f:\|f\|_{p, w}=\left(\int_{\mathbb{R}^{n}}|f(x)|^{p} w(x) \mathrm{d} x\right)^{1 / p}<\infty\right\} .
$$

And also, the weighted weak Lebesgue spaces is defined by

$$
\begin{aligned}
W L_{w}^{p}\left(\mathbb{R}^{n}\right)= & \left\{f:\|f\|_{W L_{w}^{p}}=\sup _{\lambda>0} \lambda\right. \\
& \left.\cdot w\left(\left\{x \in \mathbb{R}^{n}:|f(x)|>\lambda\right\}\right)^{1 / p}<\infty\right\} .
\end{aligned}
$$

Let us now turn to recall the definition of the weighted weak Hardy spaces. The weak Hardy spaces were first introduced in [16]. The atomic decomposition theory of weak $H^{1}$ spaces on $\mathbb{R}^{n}$ was given by Fefferman and Soria in [17]. Later, Liu established the weak $H^{p}$ spaces on homogeneous groups in [18]. In 2000, Quek and Yang introduced the weighted weak Hardy spaces $W H_{w}^{p}\left(\mathbb{R}^{n}\right)$ in [19] and established their atomic decompositions. Moreover, by using the atomic decomposition theory of $W H_{w}^{p}\left(\mathbb{R}^{n}\right)$, Quek and Yang also obtained the boundedness of $C-Z$ operators on these weighted spaces in [19]. Let $w \in A_{\infty}, 0<p \leqslant 1$, and $N=\left[n\left(q_{w} / p-1\right)\right]$. Define

$$
\begin{aligned}
\mathscr{A}_{N, w}= & \left\{\varphi \in \mathcal{S}\left(\mathbb{R}^{n}\right):\right. \\
& \sup _{x \in \mathbb{R}^{n}|\alpha| \leqslant N+1} \sup _{\left.1+|x|)^{N+n+1}\left|D^{\alpha} \varphi(x)\right| \leqslant 1\right\},}(1+
\end{aligned}
$$

where, $\alpha=\left(\alpha_{1}, \ldots, \alpha_{n}\right) \in(\mathbb{N} \cup\{0\})^{n},|\alpha|=\alpha_{1}+\cdots+\alpha_{n}$, $D^{\alpha} \varphi=\partial^{|\alpha|} \varphi /\left(\partial x_{1}^{\alpha_{1}} \cdots \partial x_{n}^{\alpha_{n}}\right)$.
For $f \in \mathcal{S}^{\prime}\left(\mathbb{R}^{n}\right)$, the grand maximal function of $f$ is defined by

$$
G_{w} f(x)=\sup _{\varphi \in \mathscr{A}_{N, w}} \sup _{|y-x|<t}\left|\left(\varphi_{t} * f\right)(y)\right| .
$$

Then, weighted weak Hardy space is defined by $W H_{w}^{p}\left(\mathbb{R}^{n}\right)=$ $\left\{f \in \mathcal{S}^{\prime}\left(\mathbb{R}^{n}\right): G_{w} f \in W L_{w}^{p}\left(\mathbb{R}^{n}\right)\right\}$. Moreover, we set $\|f\|_{W H_{w}^{p}}=$ $\left\|G_{w} f\right\|_{W L_{w}^{p}}$.

Our main results are stated as follows.

Theorem 1. Let $\Omega \in L^{2}\left(S^{n-1}\right)$ satisfying (1) and the following condition

$$
\int_{0}^{1} \frac{\omega_{2}(\delta)}{\delta^{1+\alpha}} \mathrm{d} \delta<\infty, \quad 0<\alpha \leqslant 1 .
$$

Then, for $\rho>n / 2, w \in A_{1}$, there exists a constant $C>0$ such that

$$
\left\|\mu_{\Omega, S}^{\rho}(f)\right\|_{W L_{w}^{1}} \leqslant C\|f\|_{W H_{w}^{1}} .
$$

The relationship between condition (11) and $\operatorname{Lip}_{\alpha}\left(S^{n-1}\right)$ condition is not clear up to now. We point that the conclusion of Theorem 1 still holds if we replace the condition (11) by the $\operatorname{Lip}_{\alpha}\left(S^{n-1}\right)(0<\alpha \leqslant 1)$ condition. In other words, we have the following result.

Theorem 2. Let $\Omega \in \operatorname{Lip}_{\alpha}\left(S^{n-1}\right), 0<\alpha \leqslant 1$, satisfying (1). Then, for $\rho>n / 2, w \in A_{1}$, there exists a constant $C>0$ such that

$$
\left\|\mu_{\Omega, S}^{\rho}(f)\right\|_{W L_{w}^{1}} \leqslant C\|f\|_{W H_{w}^{1}} .
$$

Theorem 3. Let $\Omega \in L^{2}\left(S^{n-1}\right)$ satisfying (1) and the following condition

$$
\int_{0}^{1} \frac{\omega_{2}(\delta)}{\delta^{1+\alpha}} \mathrm{d} \delta<\infty, \quad 0<\alpha \leqslant 1 .
$$

Then, for $\rho>n / 2, w \in A_{1}, \lambda>2$, there exists a constant $C>0$ such that

$$
\left\|\mu_{\lambda}^{*, \rho}(f)\right\|_{W L_{w}^{1}} \leqslant C\|f\|_{W H_{w}^{1}} .
$$

\section{Notations and Preliminaries}

In this section, we will introduce some notations and preliminary lemmas used in the proofs of our main theorems in the next section.

The classical $A_{p}$ weighted theory was first introduced by Muckenhoupt in the study of weighted $L^{p}$ boundedness of Hardy-Littlewood maximal functions in [20]. A weight $w$ is a locally integrable function on $\mathbb{R}^{n}$ which takes values in $(0, \infty)$ at almost everywhere. Given a ball $B$ and $\lambda>0, \lambda B$ denotes the ball with the same center as $B$ whose radius is $\lambda$ times that of $B$. We also denote the weighted measure of $E$ by $w(E)$; that is, $w(E)=\int_{E} w(x) \mathrm{d} x$. We say that $w \in A_{p}$ with $1<p<\infty$ if there exists a constant $C>0$, such that for every ball $B \subset \mathbb{R}^{n}$,

$$
\left(\frac{1}{|B|} \int_{B} w(x) \mathrm{d} x\right)\left(\frac{1}{|B|} \int_{B} w(x)^{-1 /(p-1)} \mathrm{d} x\right)^{p-1} \leqslant C .
$$


We say that $w \in A_{1}$ if

$$
\frac{1}{|B|} \int_{B} w(x) \mathrm{d} x \leqslant C \cdot \text { ess } \inf _{x \in B} w(x) \quad \text { for every ball } B \subset \mathbb{R}^{n} \text {. }
$$

A weight function $w \in A_{\infty}$ if it satisfies the $A_{p}$ condition for some $1<p<\infty$. It is well known that if $w \in A_{p}, 1<p<\infty$, then $w \in A_{r}$ for all $r>p$, and $w \in A_{q}$ for some $1<q<p$. We thus write $q_{w} \equiv \inf \left\{q>1: w \in A_{q}\right\}$ to denote the critical index of $w$.

Lemma 4 (see [21]). Let $1 \leqslant p<\infty, w \in A_{p}$. Then, for any ball $B$, there exists an absolute constant $C>0$, such that

$$
w(2 B) \leqslant C w(B) .
$$

In general, for any $\lambda>0$, we have

$$
w(\lambda B) \leqslant C \lambda^{n p} w(B),
$$

where $C$ does not depend on $B$ nor on $\lambda$.

Lemma 5 (see [19]). Let $0<p \leqslant 1, w \in A_{\infty}$. For every $f(x)$ belongs to $W H_{w}^{p}\left(\mathbb{R}^{n}\right)$, there exists a sequence of bounded measurable functions $\left\{f_{k}(x)\right\}_{k=-\infty}^{\infty}$ such that

(i) $f(x)=\sum_{k=-\infty}^{\infty} f_{k}(x)$, in $\mathcal{S}^{\prime}$,

(ii) each $f_{k}$ can be further decomposed into $f_{k}=\sum_{i} b_{i}^{k}$, where $b_{i}^{k}$ satisfies the following conditions.

(a) $\operatorname{supp}\left(b_{i}^{k}\right) \subset Q_{i}^{k}$, where $Q_{i}^{k}$ denotes the ball with center $x_{i}^{k}$ and radius $r_{i}^{k}$. Moreover,

$\sum_{i} w\left(Q_{i}^{k}\right) \leqslant C_{1} 2^{-k p}, \quad \sum_{i} \chi_{Q_{i}^{k}} \leqslant C_{1}$

where $\chi_{E}$ denotes the characteristic function of the set $E$ and $C_{1} \leqslant\|f\|_{W H_{w}^{p}}^{p}$.

(b) $\left\|b_{i}^{k}\right\|_{L^{\infty}} \leqslant C 2^{k}$, where $C>0$ is independent of $i$, $k$.

(c) $\int_{\mathbb{R}^{n}} b_{i}^{k} x^{\alpha} \mathrm{d} x=0$ for every multi-index $\alpha$ with $|\alpha| \leqslant\left[n\left(q_{w} / p-1\right)\right]$.

Conversely, if $f \in \mathcal{S}^{\prime}\left(\mathbb{R}^{n}\right)$ have a decomposition satisfying (i) and (ii), then $f \in W H_{w}^{p}\left(\mathbb{R}^{n}\right)$. Moreover, we have $\|f\|_{W H_{w}^{p}}^{p} \sim$ C.

In the end of this section, we need the following lemmas used in the next section.

Lemma 6 (see [22]). Suppose that $\Omega \in L^{2}\left(S^{n-1}\right)$ satisfies (1) and the following condition

$$
\int_{0}^{1} \frac{\omega_{2}(\delta)}{\delta}(1+|\log \delta|)^{\sigma} \mathrm{d} \delta<\infty, \quad \sigma>1,
$$

$w \in A_{p}$. Then, for $\rho>n / 2, \lambda>2$, and $f \in L^{p}\left(\mathbb{R}^{n}\right)(1<p<$ $\infty)$, there is a constant $C$ independent of $f$, such that

$$
\begin{aligned}
& \left\|\mu_{\Omega, S}^{\rho}(f)\right\|_{L_{w}^{p}} \leqslant C\|f\|_{L_{w}^{p},} \\
& \left\|\mu_{\lambda}^{*, \rho}(f)\right\|_{L^{p}} \leqslant C\|f\|_{L_{w}^{p} .}
\end{aligned}
$$

Lemma 7 (see [23]). Suppose that $\rho>0, \Omega$ is homogeneous of degree zero and satisfies the $L^{2}-D i n i$ condition. If there exists $a$ constant $0<\theta<1 / 2$ such that $|x|<\theta R$, then we have

$$
\begin{aligned}
& \left(\int_{R<|y|<2 R} \mid \frac{\Omega(y-x)}{|y-x|^{n-\rho}}-\frac{\Omega(y)}{\left.|y|^{n-\rho}\right|^{2}} \mathrm{~d} y\right)^{1 / 2} \\
& \leqslant C R^{n / 2-(n-\rho)}\left\{\frac{|x|}{R}+\int_{|x| / 2 R<\delta<|x| / R} \frac{\omega_{2}(\delta)}{\delta} \mathrm{d} \delta\right\},
\end{aligned}
$$

where the constant $C>0$ is independent of $R, x$.

\section{Proof of Main Results}

Proof of Theorem 1. In order to prove Theorem 1, it suffices to show that there exists a constant $C>0$, for any $f \in W H_{w}^{1}\left(\mathbb{R}^{n}\right)$ and $\beta>0$, such that

$$
\beta w\left(\left\{x \in \mathbb{R}^{n}:\left|\mu_{\Omega, S}^{\rho}(f)(x)\right|>\beta\right\}\right) \leqslant C\|f\|_{W H_{w}^{1}}
$$

Take $k_{0} \in \mathbb{Z}$ such that $2^{k_{0}} \leqslant \beta<2^{k_{0}}+1$; then by Lemma 5 we can write

$$
f=\sum_{k=-\infty}^{\infty} f_{k}=\sum_{k=-\infty}^{k_{0}} f_{k}+\sum_{k=k_{0}+1}^{\infty} f_{k}=: F_{1}+F_{2},
$$

where $F_{1}=\sum_{k=-\infty}^{k_{0}} \sum_{i} b_{i}^{k}, F_{2}=\sum_{k=k_{0}+1}^{\infty} \sum_{i} b_{i}^{k}$, and $\left\{b_{i}^{k}\right\}$ satisfies (a)-(c) in Lemma 5. Then, we have

$$
\begin{aligned}
& \beta w\left(\left\{x \in \mathbb{R}^{n}:\left|\mu_{\Omega, S}^{\rho}(f)(x)\right|>\beta\right\}\right) \\
& \leqslant \beta w\left(\left\{x \in \mathbb{R}^{n}:\left|\mu_{\Omega, S}^{\rho}\left(F_{1}\right)(x)\right|>\frac{\beta}{2}\right\}\right) \\
&+\beta w\left(\left\{x \in \mathbb{R}^{n}:\left|\mu_{\Omega, S}^{\rho}\left(F_{2}\right)(x)\right|>\frac{\beta}{2}\right\}\right) \\
&=: I_{1}+I_{2} .
\end{aligned}
$$

First, we claim that the following inequality holds:

$$
\left\|F_{1}\right\|_{L_{w}^{2}} \leqslant C \beta^{1 / 2}\|f\|_{W H_{w}^{1}}^{1 / 2}
$$

In fact, since $\operatorname{supp}\left(b_{i}^{k}\right) \subset Q_{i}^{k}=Q\left(x_{i}^{k}, r_{i}^{k}\right),\left\|b_{i}^{k}\right\|_{L^{\infty}} \leqslant C 2^{k}$, then it follows from Minkowski's integral inequality that

$$
\begin{aligned}
\left\|F_{1}\right\|_{L_{w}^{2}} & \leqslant C \sum_{k=-\infty}^{k_{0}} \sum_{i}\left\|b_{i}^{k}\right\|_{L^{\infty}} w\left(Q_{i}^{k}\right)^{1 / 2} \\
& \leqslant C \sum_{k=-\infty}^{k_{0}} 2^{k}\left(\sum_{i} w\left(Q_{i}^{k}\right)\right)^{1 / 2} \\
& \leqslant C \sum_{k=-\infty}^{k_{0}} 2^{k / 2}\|f\|_{W H_{w}^{1}}^{1 / 2} \\
& \leqslant C \sum_{k=-\infty}^{k_{0}} 2^{\left(k-k_{0}\right) / 2} \beta^{1 / 2}\|f\|_{W H_{w}^{1}}^{1 / 2} \leqslant C \beta^{1 / 2}\|f\|_{W H_{w}^{1}}^{1 / 2}
\end{aligned}
$$


Let us estimate $I_{1}$. By Chebyshev's inequality, Lemma 6 and (27), we have

$$
\begin{aligned}
I_{1} & \leqslant \beta \frac{4}{\beta^{2}} \int_{\mathbb{R}^{n}}\left|\mu_{\Omega, S}^{\rho}\left(F_{1}\right)(x)\right|^{2} w(x) \mathrm{d} x \\
& =C \frac{1}{\beta\left\|\mu_{\Omega, S}^{\rho}\left(F_{1}\right)\right\|_{L_{w}^{2}}^{2}} \\
& \leqslant C \frac{1}{\beta\left\|F_{1}\right\|_{L_{w}^{2}}^{2}} \\
& \leqslant C\|f\|_{W H_{w}^{1}} .
\end{aligned}
$$

Now we turn our attention to the estimate of $I_{2}$. If we set

$$
A_{k_{0}}=\bigcup_{k=k_{0}+1}^{\infty} \bigcup_{i} \widetilde{Q}_{i}^{k},
$$

where $\widetilde{Q}_{i}^{k}=Q\left(x_{i}^{k}, 8 \tau^{\left(k-k_{0}\right) / n} r_{i}^{k}\right)$ and $\tau$ is a fixed positive number such that $1<\tau<2$, therefore,

$$
\begin{aligned}
& I_{2}=\beta w\left(\left\{x \in 2 A_{k_{0}}:\left|\mu_{\Omega, S}^{\rho}\left(F_{2}\right)(x)\right|>\frac{\beta}{2}\right\}\right) \\
& I_{2}^{\prime \prime} \leqslant \beta \frac{2}{\beta} \int_{\left(2 A_{k_{0}}{ }^{c}\right.} \mu_{\Omega, S}^{\rho}\left(F_{2}\right)(x) w(x) \mathrm{d} x \\
& =C \int_{\left(2 A_{k_{0}}\right)^{c}}\left(\iint_{|y-x|<t}\left|\sum_{k=k_{0}+1}^{\infty} \sum_{i} \int_{|y-z|<t} \frac{\Omega(y-z)}{|y-z|^{n-\rho}} b_{i}^{k}(z) \mathrm{d} z\right|^{2} \frac{\mathrm{d} t \mathrm{~d} y}{t^{n+2 \rho+1}}\right)^{1 / 2} w(x) \mathrm{d} x \\
& \leqslant C \sum_{k=k_{0}+1}^{\infty} \sum_{i} \int_{\left(2 A_{k_{0}}\right)^{c}}\left(\iint_{|y-x|<t}\left|\int_{|y-z|<t} \frac{\Omega(y-z)}{|y-z|^{n-\rho}} b_{i}^{k}(z) \mathrm{d} z\right|^{2} \frac{\mathrm{d} t \mathrm{~d} y}{t^{n+2 \rho+1}}\right)^{1 / 2} w(x) \mathrm{d} x \\
& \leqslant C \sum_{k=k_{0}+1}^{\infty} \sum_{i}\left[\int_{\left(2 A_{k_{0}}\right)^{c}}\left(\iint_{\substack{y \in 4 Q_{i}^{k} \\
|y-x|<t}}\left|\int_{|y-z|<t} \frac{\Omega(y-z)}{|y-z|^{n-\rho}} b_{i}^{k}(z) \mathrm{d} z\right|^{2} \frac{\mathrm{d} t \mathrm{~d} y}{t^{n+2 \rho+1}}\right)^{1 / 2} w(x) \mathrm{d} x\right. \\
& \left.+\int_{\left(2 A_{k_{0}}\right)^{c}}\left(\iint_{\substack{y \in\left(4 \mathrm{Q}_{i}^{k}\right)^{c} \\
|y-x|<t}}\left|\int_{|y-z|<t} \frac{\Omega(y-z)}{|y-z|^{n-\rho}} b_{i}^{k}(z) \mathrm{d} z\right|^{2} \frac{\mathrm{d} t \mathrm{~d} y}{t^{n+2 \rho+1}}\right)^{1 / 2} w(x) \mathrm{d} x\right] \\
& =: C \sum_{k=k_{0}+1}^{\infty} \sum_{i}\left[I_{3}+I_{4}\right] \text {. }
\end{aligned}
$$

Since $w \in A_{1}$, then by Lemma 4 we can get

$$
\begin{aligned}
I_{2}^{\prime} & \leqslant C \beta \sum_{k=k_{0}+1}^{\infty} \sum_{i} w\left(\widetilde{Q}_{i}^{k}\right) \\
& \leqslant C \beta \sum_{k=k_{0}+1}^{\infty} \tau^{k-k_{0}} \sum_{i} w\left(Q_{i}^{k}\right) \\
& \leqslant C\|f\|_{W H_{w}^{1}} \sum_{k=k_{0}+1}^{\infty}\left(\frac{\tau}{2}\right)^{k-k_{0}} \\
& \leqslant C\|f\|_{W H_{w}^{1}} .
\end{aligned}
$$

An application of Chebyshev's inequality and Minkowski

Firstly, let us estimate $I_{3}$. As $y \in 4 Q_{i}^{k}, x \in\left(2 A_{k_{0}}\right)^{c}, z \in Q_{i}^{k}$, it is easy to see that

$$
\begin{gathered}
|y-x| \geqslant\left|x-x_{i}^{k}\right|-\left|y-x_{i}^{k}\right| \geqslant \frac{\left|x-x_{i}^{k}\right|}{2} \\
|y-z|<8 r_{i}^{k}
\end{gathered}
$$


Abstract and Applied Analysis

5

$$
\begin{aligned}
I_{3} & \leqslant C 2^{k} \int_{\left(2 A_{k_{0}}\right)^{c}}\left(\iint_{\substack{y \in 4 Q_{i}^{k} \\
\left|x-x_{i}^{k}\right| / 2 \leqslant|y-x|<t}} \mid \int_{|y-z|<t} \frac{\Omega(y-z)}{\left.\left.|y-z|^{n-\rho} \mathrm{d} z\right|^{2} \frac{\mathrm{d} t \mathrm{~d} y}{t^{n+2 \rho+1}}\right)^{1 / 2} w(x) \mathrm{d} x}\right. \\
& \leqslant C 2^{k} \int_{\left(2 A_{k_{0}}\right)^{c}}\left(\int_{y \in 4 \mathrm{Q}_{i}^{k}} \int_{\left|x-x_{i}^{k}\right| / 2}^{\infty}\left|\int_{S^{n-1}} \int_{0}^{8 r_{i}^{k}} \frac{\left|\Omega\left(z^{\prime}\right)\right|}{s^{n-\rho}} s^{n-1} \mathrm{~d} s \mathrm{~d} \sigma\left(z^{\prime}\right)\right|^{2} \frac{\mathrm{d} t \mathrm{~d} y}{t^{n+2 \rho+1}}\right)^{1 / 2} w(x) \mathrm{d} x \\
& \leqslant C 2^{k} \int_{\left(2 A_{k_{0}}\right)^{c}}\left(\int_{y \in 4 \mathrm{Q}_{i}^{k}} \int_{\left|x-x_{i}^{k}\right| / 2}^{\infty}\left(8 r_{i}^{k}\right)^{2 \rho} \frac{\mathrm{d} t \mathrm{~d} y}{t^{n+2 \rho+1}}\right)^{1 / 2} w(x) \mathrm{d} x \\
& \leqslant C 2^{k}\left(8 r_{i}^{k}\right)^{\rho} \int_{\left(2 A_{k_{0}}\right)^{c}}\left(\int_{y \in 4 \mathrm{Q}_{i}^{k}} \frac{\mathrm{d} y}{\left|x-x_{i}^{k}\right|^{n+2 \rho}}\right)^{1 / 2} w(x) \mathrm{d} x \\
& \leqslant C 2^{k}\left(8 r_{i}^{k}\right)^{\rho}\left|4 Q_{i}^{k}\right|^{1 / 2} \int_{\left(2 A_{k_{0}}\right)^{c}} \frac{w(x)}{\left|x-x_{i}^{k}\right|^{n / 2+\rho}} \mathrm{d} x .
\end{aligned}
$$

Since $x \in\left(2 A_{k_{0}}\right)^{c}, \widetilde{Q}_{i}^{k}=Q\left(x_{i}^{k}, 8 \tau^{\left(k-k_{0}\right) / n} r_{i}^{k}\right)$, then $\left|x-x_{i}^{k}\right|>$ $8 \tau^{\left(k-k_{0}\right) / n} r_{i}^{k}>8 r_{i}^{k}$. By Lemma 4 , we obtain that

$$
I_{3} \leqslant C 2^{k}\left(r_{i}^{k}\right)^{\rho+n / 2}
$$$$
\times \sum_{j=3}^{\infty} \int_{2^{j} \tau^{\left(k-k_{0}\right) / n} r_{i}^{k}<\left|x-x_{i}^{k}\right|<2^{j+1} \tau^{\left(k-k_{0}\right) / n} r_{i}^{k}} \frac{w(x)}{\left|x-x_{i}^{k}\right|^{n / 2+\rho}} \mathrm{d} x
$$$$
\leqslant C 2^{k}\left(r_{i}^{k}\right)^{\rho+n / 2}
$$$$
\times \sum_{j=3}^{\infty}\left(2^{j+1} \tau^{\left(k-k_{0}\right) / n} r_{i}^{k}\right)^{-n / 2-\rho}
$$$$
\times \int_{\left|x-x_{i}^{k}\right|<2^{j+1} \tau^{\left(k-k_{0}\right) / n} r_{i}^{k}} w(x) \mathrm{d} x
$$$$
\leqslant C 2^{k} \sum_{j=3}^{\infty} 2^{-(\rho-n / 2) j} \frac{1}{2^{(j+1) n}}\left(2^{j+1} \tau^{\left(k-k_{0}\right) / n}\right)^{n} w\left(Q_{i}^{k}\right)
$$

$$
\begin{aligned}
& \leqslant C 2^{k} \sum_{j=3}^{\infty} 2^{-(\rho-n / 2) j}\left(\tau^{\left(k-k_{0}\right) / n}\right)^{n / 2-\rho} w\left(Q_{i}^{k}\right) \\
& \leqslant C 2^{k}\left(\tau^{\left(k-k_{0}\right) / n}\right)^{n / 2-\rho} w\left(Q_{i}^{k}\right) .
\end{aligned}
$$

Noticing that $\rho>n / 2,1<\tau<2$, we have

Now we consider $I_{4}$. Write

$$
\begin{aligned}
\sum_{k=k_{0}+1}^{\infty} \sum_{i} I_{3} & \leqslant C \sum_{k=k_{0}+1}^{\infty} 2^{k}\left(\tau^{\left(k-k_{0}\right) / n}\right)^{n / 2-\rho} \sum_{i} w\left(Q_{i}^{k}\right) \\
& \leqslant C \sum_{k=k_{0}+1}^{\infty}\left(\tau^{\left(k-k_{0}\right) / n}\right)^{n / 2-\rho}\|f\|_{W H_{w}^{1}} \\
& \leqslant C\|f\|_{W H_{w}^{1}} .
\end{aligned}
$$

$$
\begin{aligned}
& I_{4} \leqslant C \int_{\left(2 A_{k_{0}}\right)^{c}}\left(\iint_{\substack{y \in\left(4 \mathrm{Q}_{i}^{k}\right)^{c} \\
|y-x|<t \\
t \leqslant\left|y-x_{i}^{k}\right|+2 r_{i}^{k}}}\left|\int_{|y-z|<t} \frac{\Omega(y-z)}{|y-z|^{n-\rho}} b_{i}^{k}(z) \mathrm{d} z\right|^{2} \frac{\mathrm{d} t \mathrm{~d} y}{t^{n+2 \rho+1}}\right)^{1 / 2} w(x) \mathrm{d} x \\
& +C \int_{\left(2 A_{k_{0}}\right)^{c}}\left(\iint_{\begin{array}{c}
y \in\left(4 Q_{i}^{k}\right)^{c} \\
|y-x|<t \\
\left|y-x_{i}^{k}\right|+2 r_{i}^{k}<t
\end{array}}\left|\int_{|y-z|<t} \frac{\Omega(y-z)}{|y-z|^{n-\rho}} b_{i}^{k}(z) \mathrm{d} z\right|^{2} \frac{\mathrm{d} t \mathrm{~d} y}{t^{n+2 \rho+1}}\right)^{1 / 2} w(x) \mathrm{d} x \\
& =: C\left(I_{41}+I_{42}\right) .
\end{aligned}
$$


Take $0<\varepsilon<\min \{1 / 2, \alpha, \rho-n / 2\}$. First we deal with $I_{41}$. As $|y-z|<t$, we have

$$
\begin{aligned}
& t>|y-z| \geqslant\left|y-x_{i}^{k}\right|-\left|x_{i}^{k}-z\right| \\
& \geqslant\left|y-x_{i}^{k}\right|-2 r_{i}^{k}, \\
& \left|x-x_{i}^{k}\right| \leqslant|x-y|+\left|y-x_{i}^{k}\right| \leqslant t+\left|y-x_{i}^{k}\right|
\end{aligned}
$$

$$
\begin{gathered}
\leqslant 2\left|y-x_{i}^{k}\right|+2 r_{i}^{k} \leqslant 3\left|y-x_{i}^{k}\right|, \\
\left|\frac{1}{\left(\left|y-x_{i}^{k}\right|-2 r_{i}^{k}\right)^{n+2 \rho}}-\frac{1}{\left(\left|y-x_{i}^{k}\right|+2 r_{i}^{k}\right)^{n+2 \rho}}\right| \\
\leqslant C \frac{r_{i}^{k}}{\left|y-x_{i}^{k}\right|^{n+2 \rho+1}} .
\end{gathered}
$$

Using the Minkowski inequality, we get that

$$
\begin{aligned}
& I_{41} \leqslant \int_{\left(2 A_{k_{0}}\right)^{c}} \int_{\mathbb{R}^{n}}\left|b_{i}^{k}(z)\right|\left(\iint_{\substack{y \in\left(4 Q_{i}^{k}\right)^{c} \\
|y-x|<t \\
t \leqslant\left|y-x_{i}^{k}\right|+2 r_{i}^{k} \\
|y-z|<t}} \frac{|\Omega(y-z)|^{2}}{|y-z|^{2 n-2 \rho}} \frac{\mathrm{d} t \mathrm{~d} y}{t^{n+2 \rho+1}}\right)^{1 / 2} \mathrm{~d} z w(x) \mathrm{d} x \\
& \leqslant \int_{\left(2 A_{k_{0}}\right)^{c}} \int_{\mathbb{R}^{n}}\left|b_{i}^{k}(z)\right|\left(\int_{\substack{y \in\left(4 Q_{i}^{k}\right)^{c} \\
\left|x-x_{i}^{k}\right| \leqslant 3\left|y-x_{i}^{k}\right|}} \frac{|\Omega(y-z)|^{2}}{|y-z|^{2 n-2 \rho}}\left(\int_{\left|y-x_{i}^{k}\right|-2 r_{i}^{k}}^{\left|y-x_{i}^{k}\right|+2 r_{i}^{k}} \frac{\mathrm{d} t}{t^{n+2 \rho+1}}\right) \mathrm{d} y\right)^{1 / 2} \mathrm{~d} z w(x) \mathrm{d} x \\
& \leqslant C \int_{\left(2 A_{k_{0}}\right)^{c}} \int_{\mathbb{R}^{n}}\left|b_{i}^{k}(z)\right| \\
& \times\left(\int_{\substack{y \in\left(4 Q_{i}^{k}\right)^{c} \\
\left|x-x_{i}^{k}\right| \leqslant 3\left|y-x_{i}^{k}\right|}} \frac{|\Omega(y-z)|^{2}}{|y-z|^{2 n-2 \rho}}\left|\frac{1}{\left(\left|y-x_{i}^{k}\right|-2 r_{i}^{k}\right)^{n+2 \rho}}-\frac{1}{\left(\left|y-x_{i}^{k}\right|+2 r_{i}^{k}\right)^{n+2 \rho}}\right| \mathrm{d} y\right)^{1 / 2} \mathrm{~d} z w(x) \mathrm{d} x \\
& \leqslant C \int_{\left(2 A_{k_{0}}\right)^{c}} \int_{\mathbb{R}^{n}}\left|b_{i}^{k}(z)\right|\left(\int_{\substack{y \in\left(4 Q_{i}^{k}\right)^{c} \\
\left|x-x_{i}^{k}\right| \leqslant 3\left|y-x_{i}^{k}\right|}} \frac{|\Omega(y-z)|^{2}}{|y-z|^{2 n-2 \rho}} \frac{r_{i}^{k}}{\left|y-x_{i}^{k}\right|^{n+2 \rho+1}} \mathrm{~d} y\right)^{1 / 2} \mathrm{~d} z w(x) \mathrm{d} x \\
& \leqslant C \int_{\left(2 A_{k_{0}}\right)^{c}} \int_{\mathbb{R}^{n}}\left|b_{i}^{k}(z)\right|\left(\int_{y \in\left(4 Q_{i}^{k}\right)^{c}} \frac{|\Omega(y-z)|^{2}}{|y-z|^{n-2 \varepsilon+1}} \frac{r_{i}^{k}}{\left|x-x_{i}^{k}\right|^{2 n+2 \varepsilon}} \mathrm{d} y\right)^{1 / 2} \mathrm{~d} z w(x) \mathrm{d} x \\
& \leqslant C\left(r_{i}^{k}\right)^{1 / 2} \int_{\left(2 A_{k_{0}}\right)^{c}} \int_{\mathbb{R}^{n}} \frac{\left|b_{i}^{k}(z)\right|}{\left|x-x_{i}^{k}\right|^{n+\varepsilon}}\left(\int_{y \in\left(4 Q_{i}^{k}\right)^{c}} \frac{|\Omega(y-z)|^{2}}{|y-z|^{n-2 \varepsilon+1}} \mathrm{~d} y\right)^{1 / 2} \mathrm{~d} z w(x) \mathrm{d} x \\
& \leqslant C\left(r_{i}^{k}\right)^{1 / 2} \int_{\left(2 A_{k_{0}}\right)^{c}} \int_{\mathbb{R}^{n}} \frac{\left|b_{i}^{k}(z)\right|}{\left|x-x_{i}^{k}\right|^{n+\varepsilon}}\left(\int_{S^{n-1}} \int_{2 r_{i}^{k}}^{\infty} \frac{\left|\Omega\left(z^{\prime}\right)\right|^{2}}{s^{2-2 \varepsilon}} \mathrm{d} s \mathrm{~d} \sigma\left(z^{\prime}\right)\right)^{1 / 2} \mathrm{~d} z w(x) \mathrm{d} x \\
& \leqslant C\left(r_{i}^{k}\right)^{\varepsilon} \int_{\left(2 A_{k_{0}}\right)^{c}} \frac{w(x)}{\left|x-x_{i}^{k}\right|^{n+\varepsilon}} \mathrm{d} x \int_{Q_{i}^{k}}\left|b_{i}^{k}(z)\right| \mathrm{d} z \\
& \leqslant C 2^{k}\left(r_{i}^{k}\right)^{\varepsilon+n} \sum_{j=3}^{\infty} \int_{2^{j} \tau^{\left(k-k_{0}\right) / n} r_{i}^{k}<\left|x-x_{i}^{k}\right|<2^{j+1} \tau^{\left(k-k_{0}\right) / n} r_{i}^{k}} \frac{w(x)}{\left|x-x_{i}^{k}\right|^{n+\varepsilon}} \mathrm{d} x \\
& \leqslant C 2^{k}\left(r_{i}^{k}\right)^{\varepsilon+n} \sum_{j=3}^{\infty}\left(2^{j+1} r_{i}^{k}\right)^{-n-\varepsilon} \tau^{\left(k-k_{0}\right)(-n-\varepsilon) / n} \int_{\left|x-x_{i}^{k}\right|<2^{j+1} \tau^{\left(k-k_{0}\right) / n_{r} k}} w(x) \mathrm{d} x
\end{aligned}
$$




$$
\begin{aligned}
& \leqslant C 2^{k} \sum_{j=3}^{\infty}\left(2^{j+1}\right)^{-n-\varepsilon} \tau^{\left(k-k_{0}\right)(-n-\varepsilon) / n} 2^{(j+1) n} \tau^{k-k_{0}} w\left(Q_{i}^{k}\right) \\
& \leqslant C 2^{k}\left[\tau^{\left(k-k_{0}\right) / n}\right]^{-\varepsilon} \sum_{j=3}^{\infty} 2^{-\varepsilon(j+1)} w\left(Q_{i}^{k}\right) .
\end{aligned}
$$

By Lemma 5, we have

$$
\begin{aligned}
& \sum_{k=k_{0}+1}^{\infty} \sum_{i} I_{41} \\
& \leqslant C \sum_{k=k_{0}+1}^{\infty} 2^{k}\left(\tau^{\left(k-k_{0}\right) / n}\right)^{-\varepsilon} \sum_{i} w\left(Q_{i}^{k}\right)
\end{aligned}
$$

$$
\begin{aligned}
& \leqslant C \sum_{k=k_{0}+1}^{\infty}\left(\tau^{\left(k-k_{0}\right) / n}\right)^{-\varepsilon}\|f\|_{W H_{w}^{1}} \\
& \leqslant C\|f\|_{W H_{w}^{1}} .
\end{aligned}
$$

Now let us consider $I_{42}$. It is easy to check that $Q_{i}^{k} \subset\{z$ : $|y-z|<t\}$ as $y \in\left(4 Q_{i}^{k}\right)^{c}, t>\left|y-x_{i}^{k}\right|+2 r_{i}^{k}$. Thus, we can obtain by the condition (c) of $b_{i}^{k}$ in Lemma 5

$$
\begin{aligned}
& I_{42}=\int_{\left(2 A_{k_{0}}\right)^{c}}\left(\iint_{\substack{y \in\left(4 \mathrm{Q}_{i}^{k}\right)^{c} \\
|y-x|<t \\
\left|y-x_{i}^{k}\right|+2 r_{i}^{k}<t}}\left|\int_{|y-z|<t} \frac{\Omega(y-z)}{|y-z|^{n-\rho}}-\frac{\Omega\left(y-x_{i}^{k}\right)}{\left|y-x_{i}^{k}\right|^{n-\rho}} b_{i}^{k}(z) \mathrm{d} z\right|^{2} \frac{\mathrm{d} t \mathrm{~d} y}{t^{n+2 \rho+1}}\right)^{1 / 2} w(x) \mathrm{d} x \\
& \leqslant \int_{\left(2 A_{k_{0}}\right)^{c}} \int_{\mathbb{R}^{n}}\left|b_{i}^{k}(z)\right|\left(\iint_{\begin{array}{c}
\max \left\{|y-x|,\left|y-x_{i}^{k}\right|+2 r_{i}^{k},|y-z|\right\}<t \\
\left|x-x_{i}^{k}\right| \leqslant 2\left|y-x_{i}^{k}\right|
\end{array}}\left|\frac{\Omega(y-z)}{|y-z|^{n-\rho}}-\frac{\Omega\left(y-x_{i}^{k}\right)}{\left|y-x_{i}^{k}\right|^{n-\rho}}\right|^{2} \frac{\mathrm{d} t \mathrm{~d} y}{t^{n+2 \rho+1}}\right)^{1 / 2} \mathrm{~d} z w(x) \mathrm{d} x \\
& +\int_{\left(2 A_{k_{0}}\right)^{c}} \int_{\mathbb{R}^{n}}\left|b_{i}^{k}(z)\right|\left(\iint_{\begin{array}{c}
\max \left\{|y-x|,\left|y-x_{i}^{k}\right|+2 r_{i}^{k},|y-z|\right\}<t \\
\left|x-x_{i}^{k}\right|>2\left|y-x_{i}^{k}\right|
\end{array}}\left|\frac{\Omega(y-z)}{|y-z|^{n-\rho}}-\frac{\Omega\left(y-x_{i}^{k}\right)}{\left|y-x_{i}^{k}\right|^{n-\rho}}\right|^{2} \frac{\mathrm{d} t \mathrm{~d} y}{t^{n+2 \rho+1}}\right)^{1 / 2} \mathrm{~d} z w(x) \mathrm{d} x \\
& =I_{42}^{\prime}+I_{42}^{\prime \prime} \text {. } \\
& I_{42}^{\prime} \leqslant \int_{\left(2 A_{k_{0}}\right)^{c}} \int_{\mathbb{R}^{n}}\left|b_{i}^{k}(z)\right|\left(\int_{\substack{y \in\left(4 Q_{i}^{k}\right)^{c} \\
\left|x-x_{i}^{k}\right| \leqslant 2\left|y-x_{i}^{k}\right|}}\left|\frac{\Omega(y-z)}{|y-z|^{n-\rho}}-\frac{\Omega\left(y-x_{i}^{k}\right)}{\left|y-x_{i}^{k}\right|^{n-\rho}}\right|^{2}\left(\int_{\left|y-x_{i}^{k}\right|+2 r_{i}^{k}}^{\infty} \frac{\mathrm{d} t}{t^{n+2 \rho+1}}\right) \mathrm{d} y\right)^{1 / 2} \mathrm{~d} z w(x) \mathrm{d} x \\
& \leqslant C \int_{\left(2 A_{k_{0}}\right)^{c}} \int_{\mathbb{R}^{n}}\left|b_{i}^{k}(z)\right|\left(\int_{\substack{y \in\left(4 Q_{i}^{k}\right)^{c} \\
\left|x-x_{i}^{k}\right| \leqslant 2\left|y-x_{i}^{k}\right|}}\left|\frac{\Omega(y-z)}{|y-z|^{n-\rho}}-\frac{\Omega\left(y-x_{i}^{k}\right)}{\left|y-x_{i}^{k}\right|^{n-\rho}}\right|^{2} \frac{1}{\left(\left|y-x_{i}^{k}\right|+2 r_{i}^{k}\right)^{n+2 \rho}} \mathrm{d} y\right)^{1 / 2} \mathrm{~d} z w(x) \mathrm{d} x \\
& \leqslant C \int_{\left(2 A_{k_{0}}\right)^{c}} \int_{\mathbb{R}^{n}} \frac{\left|b_{i}^{k}(z)\right|}{\left(\left|x-x_{i}^{k}\right|+2 r_{i}^{k}\right)^{n+\varepsilon}}\left(\int_{y \in\left(4 Q_{i}^{k}\right)^{c}}\left|\frac{\Omega(y-z)}{|y-z|^{n-\rho}}-\frac{\Omega\left(y-x_{i}^{k}\right)}{\left|y-x_{i}^{k}\right|^{n-\rho}}\right|^{2} \frac{1}{\left(\left|y-x_{i}^{k}\right|+2 r_{i}^{k}\right)^{2 \rho-n-2 \varepsilon}} \mathrm{d} y\right)^{1 / 2} \mathrm{~d} z w(x) \mathrm{d} x .
\end{aligned}
$$


By using Lemma 7, we can get

$$
\begin{aligned}
& I_{42}^{\prime} \leqslant C \int_{\left(2 A_{k_{0}}\right)^{c}} \int_{\mathbb{R}^{n}} \frac{\left|b_{i}^{k}(z)\right|}{\left(\left|x-x_{i}^{k}\right|+2 r_{i}^{k}\right)^{n+\varepsilon}} \\
& \times \sum_{j=1}^{\infty}\left(\int_{2^{j} r_{i}^{k}<\left|y-x_{i}^{k}\right|<2^{j+1} r_{i}^{k}}\left|\frac{\Omega(y-z)}{|y-z|^{n-\rho}}-\frac{\Omega\left(y-x_{i}^{k}\right)}{\left|y-x_{i}^{k}\right|^{n-\rho}}\right|^{2} \frac{1}{\left(\left|y-x_{i}^{k}\right|+2 r_{i}^{k}\right)^{2 \rho-n-2 \varepsilon}} \mathrm{d} y\right)^{1 / 2} \mathrm{~d} z w(x) \mathrm{d} x \\
& \leqslant C \int_{\left(2 A_{k_{0}}\right)^{c}} \int_{\mathbb{R}^{n}} \frac{\left|b_{i}^{k}(z)\right|}{\left(\left|x-x_{i}^{k}\right|+2 r_{i}^{k}\right)^{n+\varepsilon}} \sum_{j=1}^{\infty} \frac{1}{\left(2^{j} r_{i}^{k}+2 r_{i}^{k}\right)^{\rho-n / 2-\varepsilon}} \\
& \times\left(\int_{2^{j} r_{i}^{k}<\left|y-x_{i}^{k}\right|<2^{j+1} r_{i}^{k}}\left|\frac{\Omega(y-z)}{|y-z|^{n-\rho}}-\frac{\Omega\left(y-x_{i}^{k}\right)}{\left|y-x_{i}^{k}\right|^{n-\rho}}\right|^{2} \mathrm{~d} y\right)^{1 / 2} \mathrm{~d} z w(x) \mathrm{d} x \\
& \leqslant C \int_{\left(2 A_{k_{0}}\right)^{c}} \int_{\mathbb{R}^{n}} \frac{\left|b_{i}^{k}(z)\right|}{\left(\left|x-x_{i}^{k}\right|+2 r_{i}^{k}\right)^{n+\varepsilon}} \sum_{j=1}^{\infty} \frac{1}{\left(2^{j} r_{i}^{k}+2 r_{i}^{k}\right)^{\rho-n / 2-\varepsilon}}\left(2^{j} r_{i}^{k}\right)^{n / 2-(n-\rho)}\left\{\frac{\left|z-x_{i}^{k}\right|}{2^{j} r_{i}^{k}}+\int_{\left|z-x_{i}^{k}\right| / 2^{j+1} r_{i}^{k}}^{\left|z-x_{i}^{k}\right| / 2^{j} r_{i}^{k}} \frac{w_{2}(\delta)}{\delta} \mathrm{d} \delta\right\} \mathrm{d} z w(x) \mathrm{d} x \\
& \leqslant C \int_{\left(2 A_{k_{0}}\right)^{c}} \int_{\mathbb{R}^{n}} \frac{\left|b_{i}^{k}(z)\right|}{\left(\left|x-x_{i}^{k}\right|+2 r_{i}^{k}\right)^{n+\varepsilon}} \sum_{j=1}^{\infty}\left(2^{j} r_{i}^{k}\right)^{\varepsilon}\left\{\frac{1}{2^{j}}+\int_{\left|z-x_{i}^{k}\right| / 2^{j+1} r_{i}^{k}}^{\left|z-x_{i}^{k}\right| / 2^{j} r_{i}^{k}} \frac{w_{2}(\delta)}{\delta} \mathrm{d} \delta\right\} \mathrm{d} z w(x) \mathrm{d} x \\
& \leqslant C \int_{\left(2 A_{k_{0}}\right)^{c}} \int_{\mathbb{R}^{n}} \frac{\left|b_{i}^{k}(z)\right|}{\left(\left|x-x_{i}^{k}\right|+2 r_{i}^{k}\right)^{n+\varepsilon}} \sum_{j=1}^{\infty}\left(2^{j} r_{i}^{k}\right)^{\varepsilon} \frac{1}{2^{j}} \mathrm{~d} z w(x) \mathrm{d} x \\
& +C \int_{\left(2 A_{k_{0}}\right)^{c}} \int_{\mathbb{R}^{n}} \frac{\left|b_{i}^{k}(z)\right|}{\left(\left|x-x_{i}^{k}\right|+2 r_{i}^{k}\right)^{n+\varepsilon}} \sum_{j=1}^{\infty}\left(2^{j} r_{i}^{k}\right)^{\varepsilon} \int_{\left|z-x_{i}^{k}\right| / 2^{j+1} r_{i}^{k}}^{\left|z-x_{i}^{k}\right| / 2^{j} r_{i}^{k}} \frac{w_{2}(\delta)}{\delta} \mathrm{d} \delta \mathrm{d} z w(x) \mathrm{d} x \\
& =: C\left(U_{1}+U_{2}\right) \text {. }
\end{aligned}
$$

It is easy to see that

$$
\begin{aligned}
U_{1} \leqslant & C 2^{k}\left(r_{i}^{k}\right)^{\varepsilon+n} \\
& \times \int_{\left(A_{k_{0}}\right)^{c}} \frac{w(x)}{\left(\left|x-x_{i}^{k}\right|+2 r_{i}^{k}\right)^{n+\varepsilon}} \mathrm{d} x \sum_{j=1}^{\infty} 2^{j(\varepsilon-1)} \\
\leqslant & C 2^{k}\left(r_{i}^{k}\right)^{\varepsilon+n} \int_{\left(A_{k_{0}}\right)^{c}} \frac{w(x)}{\left(\left|x-x_{i}^{k}\right|+2 r_{i}^{k}\right)^{n+\varepsilon}} \mathrm{d} x .
\end{aligned}
$$

Using the same method as what used to deal with the inequality (39), we can obtain that

$$
U_{1} \leqslant C 2^{k}\left[\tau^{\left(k-k_{0}\right) / n}\right]^{-\varepsilon} w\left(Q_{i}^{k}\right)
$$

For $U_{2}$, we have

$$
\begin{aligned}
& U_{2} \leqslant C \int_{\left(2 A_{k_{0}}\right)^{c}} \int_{\mathbb{R}^{n}} \frac{\left|b_{i}^{k}(z)\right|}{\left(\left|x-x_{i}^{k}\right|+2 r_{i}^{k}\right)^{n+\varepsilon}} \\
& \quad \times \sum_{j=1}^{\infty} \frac{\left(2^{j} r_{i}^{k}\right)^{\varepsilon}}{2^{j \alpha}} \int_{\left|z-x_{i}^{k}\right| / 2^{j+1} r_{i}^{k}}^{\left|z-x_{i}^{k}\right| / 2^{j} r_{i}^{k}} \frac{w_{2}(\delta)}{\delta^{1+\alpha}} \mathrm{d} \delta \mathrm{d} z w(x) \mathrm{d} x \\
& \leqslant C \int_{\left(2 A_{k_{0}}\right)^{c}} \int_{\mathbb{R}^{n}} \frac{\left(r_{i}^{k}\right)^{\varepsilon}\left|b_{i}^{k}(z)\right|}{\left(\left|x-x_{i}^{k}\right|+2 r_{i}^{k}\right)^{n+\varepsilon}} \mathrm{d} z w(x) \mathrm{d} x \\
& \quad \times \sum_{j=1}^{\infty} 2^{j(\varepsilon-\alpha)} \int_{0}^{1} \frac{w_{2}(\delta)}{\delta^{1+\alpha}} \mathrm{d} \delta \\
& \leqslant C 2^{k}\left(r_{i}^{k}\right)^{\varepsilon+n} \int_{\left(2 A_{k_{0}}\right)^{c}} \frac{w(x)}{\left(\left|x-x_{i}^{k}\right|+2 r_{i}^{k}\right)^{n+\varepsilon}} \mathrm{d} x \\
& \leqslant C 2^{k}\left[\tau^{\left(k-k_{0}\right) / n}\right]^{-\varepsilon} w\left(Q_{i}^{k}\right) .
\end{aligned}
$$


Hence, by the inequalities (44) and (45), we have

$$
I_{42}^{\prime} \leqslant C\left(U_{1}+U_{2}\right) \leqslant C 2^{k}\left[\tau^{\left(k-k_{0}\right) / n}\right]^{-\varepsilon} w\left(Q_{i}^{k}\right) .
$$

Now we give the estimate for $I_{42}^{\prime \prime}$. Since $\left|x-x_{i}^{k}\right|>2\left|y-x_{i}^{k}\right|$, $t>\left|y-x_{i}^{k}\right|+2 r_{i}^{k}$, then

$$
t>|x-y| \geqslant\left|x-x_{i}^{k}\right|-\left|y-x_{i}^{k}\right| \geqslant \frac{\left|x-x_{i}^{k}\right|}{2} .
$$

Thus,

$$
\begin{aligned}
& I_{42}^{\prime \prime} \leqslant \int_{\left(2 A_{k_{0}}\right)^{c}} \int_{\mathbb{R}^{n}}\left|b_{i}^{k}(z)\right| \\
& \times\left(\iint_{\max \left\{|y-x|,\left|y-x_{i}^{k}\right|+2 r_{i}^{k},|y-z|\right\}<t}^{y \in\left(4 Q_{i}^{k} c\right.} \frac{1}{\left(\left|y-x_{i}^{k}\right|+2 r_{i}^{k}\right)^{2 \rho-n-2 \varepsilon}}\left|\frac{\Omega(y-z)}{|y-z|^{n-\rho}}-\frac{\Omega\left(y-x_{i}^{k}\right)}{\left|y-x_{i}^{k}\right|^{n-\rho}}\right|^{2} \frac{\mathrm{d} t \mathrm{~d} y}{t^{2 n+2 \varepsilon+1}}\right)^{1 / 2} \mathrm{~d} z w(x) \mathrm{d} x \\
& \leqslant \int_{\left(2 A_{k_{0}}\right)^{c}} \int_{\mathbb{R}^{n}}\left|b_{i}^{k}(z)\right|\left(\int_{y \in\left(4 Q_{i}^{k}\right)^{c}} \frac{1}{\left(\left|y-x_{i}^{k}\right|+2 r_{i}^{k}\right)^{2 \rho-n-2 \varepsilon}}\left|\frac{\Omega(y-z)}{|y-z|^{n-\rho}}-\frac{\Omega\left(y-x_{i}^{k}\right)}{\left|y-x_{i}^{k}\right|^{n-\rho}}\right|^{2}\left(\int_{\left|x-x_{i}^{k}\right| / 2}^{\infty} \frac{\mathrm{d} t}{t^{2 n+2 \varepsilon+1}}\right) \mathrm{d} y\right)^{1 / 2} \mathrm{~d} z w(x) \mathrm{d} x \\
& \leqslant \int_{\left(2 A_{k_{0}}\right)^{c}} \int_{\mathbb{R}^{n}}\left|b_{i}^{k}(z)\right|\left(\int_{y \in\left(4 Q_{i}^{k}\right)^{c}} \frac{1}{\left(\left|y-x_{i}^{k}\right|+2 r_{i}^{k}\right)^{2 \rho-n-2 \varepsilon}}\left|\frac{\Omega(y-z)}{|y-z|^{n-\rho}}-\frac{\Omega\left(y-x_{i}^{k}\right)}{\left|y-x_{i}^{k}\right|^{n-\rho}}\right|^{2} \frac{1}{\left|x-x_{i}^{k}\right|^{2 n+2 \varepsilon}} \mathrm{d} y\right)^{1 / 2} \mathrm{~d} z w(x) \mathrm{d} x \\
& \leqslant \int_{\left(2 A_{k_{0}}\right)^{c}} \int_{\mathbb{R}^{n}} \frac{\left|b_{i}^{k}(z)\right|}{\left|x-x_{i}^{k}\right|^{n+\varepsilon}}\left(\int_{y \in\left(4 Q_{i}^{k}\right)^{c}} \frac{1}{\left(\left|y-x_{i}^{k}\right|+2 r_{i}^{k}\right)^{2 \rho-n-2 \varepsilon}}\left|\frac{\Omega(y-z)}{|y-z|^{n-\rho}}-\frac{\Omega\left(y-x_{i}^{k}\right)}{\left|y-x_{i}^{k}\right|^{n-\rho}}\right|^{2} \mathrm{~d} y\right)^{1 / 2} \mathrm{~d} z w(x) \mathrm{d} x .
\end{aligned}
$$

Repeating this process which is similar to the one of estimating $I_{42}^{\prime}$ (from (42) to (46)), we may have

$$
I_{42}^{\prime \prime} \leqslant C 2^{k}\left[\tau^{\left(k-k_{0}\right) / n}\right]^{-\varepsilon} w\left(Q_{i}^{k}\right)
$$

Thus by (36) and (40), we get

$$
\begin{aligned}
I_{2} & =I_{2}^{\prime}+I_{2}^{\prime \prime} \\
& \leqslant I_{2}^{\prime}+C \sum_{k=k_{0}+1}^{\infty} \sum_{i}\left(I_{3}+I_{4}\right) \\
& \leqslant I_{2}^{\prime}+C \sum_{k=k_{0}+1}^{\infty} \sum_{i}\left(I_{3}+I_{41}+I_{42}\right) \\
& \leqslant I_{2}^{\prime}+C \sum_{k=k_{0}+1}^{\infty} \sum_{i}\left(I_{3}+I_{41}+I_{42}^{\prime}+I_{42}^{\prime \prime}\right) \\
& \leqslant C\|f\|_{W H_{w}^{1}} \cdot
\end{aligned}
$$

This completes the proof of Theorem 1.
Proof of Theorem 2. Combining the idea of proving Theorem 1 with the similar steps as in [12] and the following inequalities

$$
\begin{aligned}
\left|\frac{\Omega(y-z)}{|y-z|^{n-\rho}}-\frac{\Omega(y-z)}{\left|y-x_{i}^{k}\right|^{n-\rho}}\right| & \leqslant C\left|\frac{1}{|y-z|^{n-\rho}}-\frac{1}{\left|y-x_{i}^{k}\right|^{n-\rho}}\right| \\
& \leqslant C \frac{r_{i}^{k}}{\left|y-x_{i}^{k}\right|^{n-\rho+1}}, \\
\left|\Omega(y-z)-\Omega\left(y-x_{i}^{k}\right)\right| & \leqslant C\left|\frac{y-z}{|y-z|}-\frac{y-x_{i}^{k}}{\left|y-x_{i}^{k}\right|}\right|^{\alpha} \\
& \leqslant C \frac{\left(r_{i}^{k}\right)^{\alpha}}{\left|y-x_{i}^{k}\right|^{\alpha}}
\end{aligned}
$$

it is not difficult to get the proof of Theorem 2. We omit the details here.

Proof of Theorem 3. We follow the strategy of the proof of Theorem 1. It suffices to show that there exists a constant $C>0$, such that, for any $f \in W H_{w}^{1}\left(\mathbb{R}^{n}\right), \beta>0$,

$$
\beta w\left(\left\{x \in \mathbb{R}^{n}:\left|\mu_{\lambda}^{*, \rho}(f)(x)\right|>\beta\right\}\right) \leqslant C\|f\|_{W H_{w}^{1}} .
$$


Take $k_{0} \in \mathbb{Z}$ such that $2^{k_{0}} \leqslant \beta<2^{k_{0}}+1$, we have

$$
\begin{aligned}
\beta w(\{x \in & \left.\left.\mathbb{R}^{n}:\left|\mu_{\lambda}^{*, \rho}(f)(x)\right|>\beta\right\}\right) \\
\leqslant & \beta w\left(\left\{x \in \mathbb{R}^{n}:\left|\mu_{\lambda}^{*, \rho}\left(F_{1}\right)(x)\right|>\frac{\beta}{2}\right\}\right) \\
& +\beta w\left(\left\{x \in \mathbb{R}^{n}:\left|\mu_{\lambda}^{*, \rho}\left(F_{2}\right)(x)\right|>\frac{\beta}{2}\right\}\right) \\
= & : J_{1}+J_{2},
\end{aligned}
$$

where the notations $F_{1}, F_{2}$ are the same as in the proof of Theorem 1. Using the same method of the proof of Theorem 1 , we can get

$$
\begin{aligned}
J_{1} & \leqslant 4 \beta^{-1} \int_{\mathbb{R}^{n}}\left|\mu_{\lambda}^{*, \rho}\left(F_{1}\right)(x)\right| w(x) \mathrm{d} x \\
& \leqslant C \beta^{-1}\left\|\mu_{\lambda}^{*, \rho}\left(F_{1}\right)\right\|_{L_{w}^{2}}^{2} \\
& \leqslant C \beta^{-1}\left\|F_{1}\right\|_{L_{w}^{2}}^{2} \\
& \leqslant C\|f\|_{W H_{w}^{1}} .
\end{aligned}
$$

$$
\begin{aligned}
& J_{2}^{\prime} \leqslant 2 \beta \sum_{k=k_{0}+1}^{\infty} \sum_{i} w\left(\widetilde{Q}_{i}^{k}\right) \leqslant C \beta \sum_{k=k_{0}+1}^{\infty} \tau^{k-k_{0}} \sum_{i} w\left(Q_{i}^{k}\right) \leqslant C \sum_{k=k_{0}+1}^{\infty}\left(\frac{\tau}{2}\right)^{k-k_{0}}\|f\|_{W H_{w}^{1}} \leqslant C\|f\|_{W H_{w}^{1}} . \\
& J_{2}^{\prime \prime} \leqslant \beta \frac{2}{\beta} \int_{\left(2 A_{k_{0}}\right)^{c}} \mu_{\lambda}^{*, \rho}\left(F_{2}\right)(x) w(x) \mathrm{d} x \\
& =C \int_{\left(2 A_{k_{0}}\right)^{c}}\left(\left.\iint_{\mathbb{R}_{+}^{n+1}}\left(\frac{t}{t+|x-y|}\right)^{\lambda n} \sum_{k=k_{0}+1}^{\infty} \sum_{i} \int_{|y-z|<t} \frac{\Omega(y-z)}{|y-z|^{n-\rho}} b_{i}^{k}(z) \mathrm{d} z\right|^{2} \frac{\mathrm{d} t \mathrm{~d} y}{t^{n+2 \rho+1}}\right)^{1 / 2} w(x) \mathrm{d} x
\end{aligned}
$$

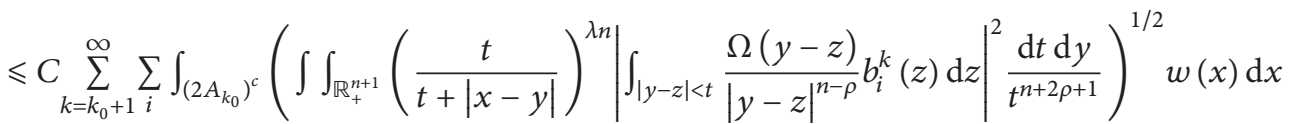

$$
\begin{aligned}
& \leqslant C \sum_{k=k_{0}+1}^{\infty} \sum_{i} \int_{\left(2 A_{k_{0}}\right)^{c}}\left(J_{3}+J_{4}\right) w(x) \mathrm{d} x,
\end{aligned}
$$

where $\widetilde{Q}_{i}^{k}=Q\left(x_{i}^{k}, 8 \tau^{\left(k-k_{0}\right) / n} r_{i}^{k}\right), \tau$ is a fixed positive number such that $1<\tau<2$; thus,

Noting that $w \in A_{2}$, then by Lemmas 4 and 5 , we have

where

$$
\begin{aligned}
& J_{3}=\left(\iint_{|y-x|<t}\left(\frac{t}{t+|x-y|}\right)^{\lambda n}\left|\int_{|y-z|<t} \frac{\Omega(y-z)}{|y-z|^{n-\rho}} b_{i}^{k}(z) \mathrm{d} z\right|^{2} \frac{\mathrm{d} t \mathrm{~d} y}{t^{n+2 \rho+1}}\right)^{1 / 2}, \\
& J_{4}=\left(\iint_{|y-x| \geqslant t}\left(\frac{t}{t+|x-y|}\right)^{\lambda n}\left|\int_{|y-z|<t} \frac{\Omega(y-z)}{|y-z|^{n-\rho}} b_{i}^{k}(z) \mathrm{d} z\right|^{2} \frac{\mathrm{d} t \mathrm{~d} y}{t^{n+2 \rho+1}}\right)^{1 / 2} .
\end{aligned}
$$

Similarly as in the proof of Theorem 1 , for $x \in\left(2 A_{k_{0}}\right)^{c}$, if the integration domains of $J_{4}$ is $y \in 4 Q_{i}^{k},|y-x| \geqslant t$, then we denote it by $J_{41}$. If $y \in\left(4 Q_{i}^{k}\right)^{c},|y-x| \geqslant t$, we denote $J_{4}$ by $J_{42}$. Moreover, if $t \leqslant\left|y-x_{i}^{k}\right|+2 r_{i}^{k}$ in the integration domains 
of $J_{42}$, we denote it by $J_{5}$, otherwise denote it by $J_{6}$. Further, we divide $J_{5}$ again by the integration domains; namely, if $\mid x-$ $x_{i}^{k}|\leqslant 2| y-x_{i}^{k} \mid$, we denote it by $J_{51}$, otherwise denote it by $J_{52}$. Now, we are in a position to give the estimates of $J_{3}, J_{41}$, $J_{51}, J_{52}, J_{6}$, respectively. First, we take $0<\varepsilon<\min \{1 / 2, \rho-$ $n / 2, \alpha,(\lambda-2) n / 2\}$ in the whole proof of Theorem 2 . Obviously,

$$
\begin{aligned}
& \int_{\left(2 A_{k_{0}}\right)^{c}} J_{3} w(x) \mathrm{d} x \\
& \leqslant \int_{\left(2 A_{k_{0}}\right)^{c}}\left(\iint_{|y-x|<t} \mid \int_{|y-z|<t} \frac{\Omega(y-z)}{|y-z|^{n-\rho}}\right. \\
& \left.\quad \times\left. b_{i}^{k}(z) \mathrm{d} z\right|^{2} \frac{\mathrm{d} t \mathrm{~d} y}{t^{n+2 \rho+1}}\right)^{1 / 2} w(x) \mathrm{d} x .
\end{aligned}
$$

By the proof of Theorem 1, we have

$$
\sum_{k=k_{0}+1}^{\infty} \sum_{i} \int_{\left(2 A_{k_{0}}\right)^{c}} J_{3} w(x) \mathrm{d} x \leqslant C\|f\|_{W H_{w}^{1}} .
$$

Notice that if $y \in 4 Q_{i}^{k}, x \in\left(2 A_{k_{0}}\right)^{c},|y-x| \geqslant t, z \in Q_{i}^{k}$, it is easy to check that

$\left(\mathrm{a}^{\prime}\right)|y-x| \geqslant\left|x-x_{i}^{k}\right|-\left|y-x_{i}^{k}\right| \geqslant\left|x-x_{i}^{k}\right| / 2,|y-z|<8 r_{i}^{k}$. If $y \in\left(4 Q_{i}^{k}\right)^{c}, x \in\left(2 A_{k_{0}}\right)^{c},|y-x| \geqslant t, z \in Q_{i}^{k}$, then

$\left(\mathrm{b}^{\prime}\right)|y-z| \sim\left|y-x_{i}^{k}\right|,\left|y-x_{i}^{k}\right|-2 r_{i}^{k} \leqslant t \leqslant\left|y-x_{i}^{k}\right|+2 r_{i}^{k}$, for $t \leqslant\left|y-x_{i}^{k}\right|+2 r_{i}^{k}$;

(c') $\left|1 /\left(\left|y-x_{i}^{k}\right|-2 r_{i}^{k}\right)^{n+2 \rho}-1 /\left(\left|y-x_{i}^{k}\right|+2 r_{i}^{k}\right)^{n+2 \rho}\right| \leqslant$ $C\left(r_{i}^{k} /\left|y-x_{i}^{k}\right|^{n+2 \rho+1}\right)$;

$\left(\mathrm{d}^{\prime}\right)|y-x| \geqslant\left|x-x_{i}^{k}\right|-\left|y-x_{i}^{k}\right| \geqslant\left|x-x_{i}^{k}\right| / 2$, for $\left|x-x_{i}^{k}\right| \geqslant$ $2\left|y-x_{i}^{k}\right|$

$\left(\mathrm{e}^{\prime}\right) t+|x-y| \geqslant t+\left|x-x_{i}^{k}\right|-\left|y-x_{i}^{k}\right| \geqslant\left|x-x_{i}^{k}\right|+2 r_{i}^{k}$, for $t>\left|y-x_{i}^{k}\right|+2 r_{i}^{k}$.

Now, let us estimate $J_{41}$. By the fact $\left(\mathrm{a}^{\prime}\right)$ and the Minkowski inequality, we have

$$
\begin{aligned}
& J_{41} \leqslant\left(\iint_{\substack{y \in 4 \mathrm{Q}_{i}^{k} \\
|y-x|>\left|x-x_{i}^{k}\right| / 2 \\
|y-x| \geqslant t}}\left(\frac{t}{t+|x-y|}\right)^{2 n+2 \varepsilon}\left|\int_{\substack{|y-z|<8 r_{i}^{k} \\
|y-z|<t}} \frac{\Omega(y-z)}{|y-z|^{n-\rho}} b_{i}^{k}(z) \mathrm{d} z\right|^{2} \frac{\mathrm{d} t \mathrm{~d} y}{t^{n+2 \rho+1}}\right)^{1 / 2} \\
& \leqslant C 2^{k} \int_{Q_{i}^{k}}\left(\iint_{\substack{y \in 4 Q_{i}^{k} \\
|y-x|>\left|x-x_{i}^{k}\right| / 2 \\
|y-z|<8 r_{i}^{k},|y-z|<t \\
|y-x| \geqslant t}}\left(\frac{t}{t+\left|x-x_{i}^{k}\right| / 2}\right)^{2 n+2 \varepsilon} \frac{|\Omega(y-z)|^{2}}{|y-z|^{2 n-2 \rho}} \frac{\mathrm{d} t \mathrm{~d} y}{t^{n+2 \rho+1}}\right)^{1 / 2} \mathrm{~d} z \\
& \leqslant C 2^{k}\left(\frac{1}{\left|x-x_{i}^{k}\right| / 2}\right)^{n+\varepsilon / 2} \int_{Q_{i}^{k}}\left(\int_{\substack{y \in 4 Q_{i}^{k} \\
|y-x|>\left|x-x_{i}^{k}\right| / 2 \\
|y-z|<8 r_{i}^{k}}} \frac{|\Omega(y-z)|^{2}}{|y-z|^{2 n-2 \rho}} \int_{0}^{|y-x|} \frac{t^{2 n+2 \varepsilon}}{\left|x-x_{i}^{k}\right|^{\varepsilon}|y-z|^{2 \rho-n-\varepsilon} t^{2 n+\varepsilon+1}} \mathrm{~d} t \mathrm{~d} y\right)^{1 / 2} \mathrm{~d} z \\
& \leqslant C 2^{k} \frac{1}{\left|x-x_{i}^{k}\right|^{n+\varepsilon / 2}} \int_{Q_{i}^{k}}\left(\int_{\substack{y \in 4 Q_{i}^{k} \\
|y-x|>\left|x-x_{i}^{k}\right| / 2 \\
|y-z|<8 r_{i}^{k}}} \frac{|\Omega(y-z)|^{2}|y-x|^{\varepsilon}}{|y-z|^{n-\varepsilon}\left|x-x_{i}^{k}\right|^{\varepsilon}} \mathrm{d} y\right)^{1 / 2} \mathrm{~d} z \\
& \leqslant C 2^{k} \frac{1}{\left|x-x_{i}^{k}\right|^{n+\varepsilon / 2}} \int_{Q_{i}^{k}}\left(\int_{|y-z|<8 r_{i}^{k}} \frac{|\Omega(y-z)|^{2}}{|y-z|^{n-\varepsilon}} \mathrm{d} y\right)^{1 / 2} \mathrm{~d} z \\
& \leqslant C 2^{k} \frac{1}{\left|x-x_{i}^{k}\right|^{n+\varepsilon / 2}} \int_{Q_{i}^{k}}\left(\int_{S^{n-1}} \int_{0}^{8 r_{i}^{k}} \frac{\left|\Omega\left(y^{\prime}\right)\right|^{2}}{|s|^{n-\varepsilon}} s^{n-1} \mathrm{~d} s \mathrm{~d} \sigma\left(y^{\prime}\right)\right)^{1 / 2} \mathrm{~d} z
\end{aligned}
$$




$$
\begin{aligned}
& \leqslant C 2^{k} \frac{1}{\left|x-x_{i}^{k}\right|^{n+\varepsilon / 2}} \int_{Q_{i}^{k}}\left(8 r_{i}^{k}\right)^{\varepsilon / 2} \mathrm{~d} z \\
& \leqslant C 2^{k} \frac{\left(r_{i}^{k}\right)^{n+\varepsilon / 2}}{\left|x-x_{i}^{k}\right|^{n+\varepsilon / 2}} .
\end{aligned}
$$

As for $J_{51}$, notice that $y \in\left(4 Q_{i}^{k}\right)^{c}, x \in\left(2 A_{k_{0}}\right)^{c},|y-x| \geqslant$ $t, z \in Q_{i}^{k}$, using the Minkowski inequality and the previous facts $\left(b^{\prime}\right)$ and $\left(c^{\prime}\right)$, we have

$$
\begin{aligned}
& J_{51} \leqslant \int_{Q_{i}^{k}}\left|b_{i}^{k}(z)\right| \\
& \times\left[\int_{\substack{y \in\left(4 \mathrm{Q}_{i}^{k}\right)^{c} \\
\left|x-x_{i}^{k}\right| \leqslant 2\left|y-x_{i}^{k}\right|}}\left(\int_{\left|y-x_{i}^{k}\right|-2 r_{i}^{k}}^{\left|y-x_{i}^{k}\right|+2 r_{i}^{k}} \frac{\mathrm{d} t}{t^{n+2 \rho+1}}\right)\right. \\
& \left.\times \frac{|\Omega(y-z)|^{2}}{|y-z|^{2 n-2 \rho}} \mathrm{d} y\right]^{1 / 2} \mathrm{~d} z \\
& \leqslant C 2^{k} \int_{Q_{i}^{k}}\left(\int_{\substack{y \in\left(4 Q_{i}^{k}\right)^{c} \\
\left|x-x_{i}^{k}\right| \leqslant 2\left|y-x_{i}^{k}\right|}} \frac{|\Omega(y-z)|^{2}}{|y-z|^{2 n-2 \rho}}\right. \\
& \left.\times \frac{r_{i}^{k}}{\left|y-x_{i}^{k}\right|^{n+2 \rho+1}} \mathrm{~d} y\right)^{1 / 2} \mathrm{~d} z \\
& \leqslant C 2^{k} \\
& \times \int_{Q_{i}^{k}}\left(\int_{\substack{y \in\left(4 Q_{i}^{k}\right)^{c} \\
\left|x-x_{i}^{k}\right| \leqslant 2\left|y-x_{i}^{k}\right|}} \frac{|\Omega(y-z)|^{2}}{|y-z|^{2 n-2 \rho}}\right.
\end{aligned}
$$

$$
\left.\times \frac{r_{i}^{k}}{\left|y-x_{i}^{k}\right|^{2 \rho-n+1-2 \varepsilon}\left|y-x_{i}^{k}\right|^{2 n+2 \varepsilon}} \mathrm{d} y\right)^{1 / 2} \mathrm{~d} z
$$$$
\leqslant C 2^{k} \frac{\left(r_{i}^{k}\right)^{1 / 2}}{\left|x-x_{i}^{k}\right|^{n+\varepsilon}}
$$$$
\times \int_{Q_{i}^{k}}\left(\int_{y \in\left(4 Q_{i}^{k}\right)^{c}} \frac{|\Omega(y-z)|^{2}}{|y-z|^{n+1-2 \varepsilon}} \mathrm{d} y\right)^{1 / 2} \mathrm{~d} z
$$$$
\leqslant C 2^{k} \frac{\left(r_{i}^{k}\right)^{n+1 / 2}}{\left|x-x_{i}^{k}\right|^{n+\varepsilon}}
$$$$
\times\left(\int_{S^{n-1}} \int_{4 r_{i}^{k}}^{\infty} \frac{\left|\Omega\left(y^{\prime}\right)\right|^{2}}{s^{2-2 \varepsilon}} \mathrm{d} s \mathrm{~d} \sigma\left(y^{\prime}\right)\right)^{1 / 2}
$$$$
\leqslant C 2^{k} \frac{\left(r_{i}^{k}\right)^{n+\varepsilon}}{\left|x-x_{i}^{k}\right|^{n+\varepsilon}} .
$$

Now we consider $J_{52}$. By the fact $\left(\mathrm{d}^{\prime}\right)$ and $t /(t+|x-y|)<t$, we have

$$
\begin{aligned}
J_{52} & \leqslant \int_{Q_{i}^{k}}\left|b_{i}^{k}(z)\right|\left[\int_{\substack{y \in\left(4 Q_{i}^{k}\right)^{c} \\
\left|x-x_{i}^{k}\right| \geqslant 2\left|y-x_{i}^{k}\right|}}\left(\int_{\left|y-x_{i}^{k}\right|-2 r_{i}^{k}}^{\left|y-x_{i}^{k}\right|+2 r_{i}^{k}}\left(\frac{t}{t+|x-y|}\right)^{2 n+2 \varepsilon} \frac{\mathrm{d} t}{t^{n+2 \rho+1}}\right) \frac{|\Omega(y-z)|^{2}}{|y-z|^{2 n-2 \rho}} \mathrm{d} y\right]^{1 / 2} \mathrm{~d} z \\
& \leqslant C 2^{k} \int_{Q_{i}^{k}}\left(\int_{\substack{y \in\left(4 Q_{i}^{k}\right)^{c} \\
\left|x-x_{i}^{k}\right| \geqslant 2\left|y-x_{i}^{k}\right|}}\left(\int_{\left|y-x_{i}^{k}\right|-2 r_{i}^{k}}^{\left|y-x_{i}^{k}\right|+2 r_{i}^{k}} \frac{t^{n+2 \varepsilon-2 \rho-1}}{|x-y|^{2 n+2 \varepsilon}} \mathrm{d} t\right) \frac{|\Omega(y-z)|^{2}}{|y-z|^{2 n-2 \rho}} \mathrm{d} y\right)^{1 / 2} \mathrm{~d} z \\
& \leqslant C 2^{k} \int_{Q_{i}^{k}}\left(\int_{\substack{y \in\left(4 Q_{i}^{k}\right)^{c} \\
\left|x-x_{i}^{k}\right| \geqslant 2\left|y-x_{i}^{k}\right|}} \frac{|\Omega(y-z)|^{2}}{|x-y|^{2 n+2 \varepsilon}|y-z|^{2 n-2 \rho}} \frac{r_{i}^{k}}{\left|y-x_{i}^{k}\right|^{2 \rho-n-2 \varepsilon+1}} \mathrm{~d} y\right)^{1 / 2} \mathrm{~d} z
\end{aligned}
$$




$$
\begin{aligned}
& \leqslant C 2^{k} \int_{Q_{i}^{k}}\left(\int_{y \in\left(4 Q_{i}^{k}\right)^{c}} \frac{|\Omega(y-z)|^{2}}{|y-z|^{n-2 \varepsilon+1}} \frac{r_{i}^{k}}{\left|x-x_{i}^{k}\right|^{2 n+2 \varepsilon}} \mathrm{d} y\right)^{1 / 2} \mathrm{~d} z \\
& \leqslant C 2^{k} \frac{\left(r_{i}^{k}\right)^{n+\varepsilon}}{\left|x-x_{i}^{k}\right|^{n+\varepsilon}} .
\end{aligned}
$$

For $y \in\left(4 Q_{i}^{k}\right)^{c}$, since $t>\left|y-x_{i}^{k}\right|+2 r_{i}^{k}$, then we have $Q_{i}^{k} \subset$ $\{z:|y-z|<t\}$. Thus, by the cancellation of $b_{i}^{k}$, we have $\int_{|y-z|<t} b_{i}^{k}(z) \mathrm{d} z=0$. By using the fact $\left(\mathrm{e}^{\prime}\right)$, we get

$$
\begin{aligned}
& J_{6}=\left(\iint_{\substack{y \in\left(4 \mathrm{Q}_{i}^{k}\right)^{c} \\
\left|y-x_{i}^{k}\right|+2 r_{i}^{k}<t \\
|y-x| \geqslant t}}\left(\frac{t}{t+|x-y|}\right)^{\lambda n}\left|\int_{|y-z|<t}\left(\frac{|\Omega(y-z)|}{|y-z|^{n-\rho}}-\frac{\left|\Omega\left(y-x_{i}^{k}\right)\right|}{\left|y-x_{i}^{k}\right|^{n-\rho}}\right) b_{i}^{k}(z) \mathrm{d} z\right|^{2} \frac{\mathrm{d} y \mathrm{~d} t}{t^{n+2 \rho+1}}\right)^{1 / 2} \\
& \leqslant \int_{\mathbb{R}^{n}}\left|b_{i}^{k}(z)\right|\left(\iint_{\substack{y \in\left(4 \mathrm{Q}_{i}^{k}\right)^{c} \\
\left|y-x_{i}^{k}\right|+2 r_{i}^{k}<t \\
|y-x| \geqslant t \\
|y-z|<t}} \frac{t^{\lambda n}}{(t+|x-y|)^{2 n+2 \varepsilon}} \frac{1}{(t+|x-y|)^{\lambda n-2 n-2 \varepsilon}}\left|\frac{|\Omega(y-z)|}{|y-z|^{n-\rho}}-\frac{\left|\Omega\left(y-x_{i}\right)^{k}\right|}{\left|y-x_{i}^{k}\right|^{n-\rho}}\right|^{2} \frac{\mathrm{d} y \mathrm{~d} t}{t^{n+2 \rho+1}}\right)^{1 / 2} \mathrm{~d} z \\
& \leqslant C \int_{Q_{i}^{k}} \frac{\left|b_{i}^{k}(z)\right|}{\left(\left|x-x_{i}^{k}\right|+2 r_{i}^{k}\right)^{n+\varepsilon}}\left(\iint_{\substack{y \in\left(4 Q_{i}^{k}\right)^{c} \\
\left|y-x_{i}^{k}\right|+2 r_{i}^{k}<t \\
|y-x| \geqslant t \\
|y-z|<t}} \frac{t^{\lambda n}}{(t+|x-y|)^{\lambda n-2 n-2 \varepsilon}} \mid \frac{|\Omega(y-z)|}{|y-z|^{n-\rho}}-\frac{\left|\Omega\left(y-x_{i}^{k}\right)\right|^{2}}{\left|y-x_{i}^{k}\right|^{n-\rho}} \frac{\mathrm{d} y \mathrm{~d} t}{t^{n+2 \rho+1}}\right)^{1 / 2} \mathrm{~d} z \\
& \leqslant C \int_{Q_{i}^{k}} \frac{\left|b_{i}^{k}(z)\right|}{\left(\left|x-x_{i}^{k}\right|+2 r_{i}^{k}\right)^{n+\varepsilon}} \\
& \times\left[\int_{\substack{y \in\left(4 \mathrm{Q}_{i}^{k}\right)^{c} \\
|y-x|>\left|y-x_{i}^{k}\right|+2 r_{i}^{k}}}\left|\frac{|\Omega(y-z)|}{|y-z|^{n-\rho}}-\frac{\left|\Omega\left(y-x_{i}^{k}\right)\right|}{\left|y-x_{i}^{k}\right|^{n-\rho}}\right|^{2}\left(\int_{\left|y-x_{i}^{k}\right|+2 r_{i}^{k}}^{|y-x|} \frac{t^{\lambda n-2 n-2 \varepsilon}}{(t+|x-y|)^{\lambda n-2 n-2 \varepsilon} t^{2 \rho-n+1-2 \varepsilon}} \mathrm{d} t\right) \mathrm{d} y\right]^{1 / 2} \mathrm{~d} z .
\end{aligned}
$$

Noting that $0<\varepsilon<\rho-n / 2$, we have $2 \rho-n-2 \varepsilon>0$. Hence

$$
\begin{aligned}
& \int_{\left|y-x_{i}^{k}\right|+2 r_{i}^{k}}^{|y-x|} \frac{1}{t^{2 \rho-n+1-2 \varepsilon}} \mathrm{d} t \\
& \quad \leqslant \int_{\left|y-x_{i}^{k}\right|+2 r_{i}^{k}}^{\infty} \frac{1}{t^{2 \rho-n+1-2 \varepsilon}} \mathrm{d} t \\
& \leqslant C \frac{1}{\left(\left|y-x_{i}^{k}\right|+2 r_{i}^{k}\right)^{2 \rho-n-2 \varepsilon}} .
\end{aligned}
$$

$$
\begin{aligned}
& \text { Thus, } \\
& \begin{aligned}
J_{6} \leqslant & C \int_{Q_{i}^{k}} \frac{\left|b_{i}^{k}(z)\right|}{\left(\left|x-x_{i}^{k}\right|+2 r_{i}^{k}\right)^{n+\varepsilon}} \\
& \times\left(\int_{y \in\left(4 Q_{i}^{k}\right)^{c}} \frac{|\Omega(y-z)|}{|y-z|^{n-\rho}}-\left.\frac{\left|\Omega\left(y-x_{i}^{k}\right)\right|^{2}}{\left|y-x_{i}^{k}\right|^{n-\rho}}\right|^{1 / 2}\right. \\
& \left.\times \frac{1}{\left|y-x_{i}^{k}\right|^{2 \rho-n-2 \varepsilon}} \mathrm{d} y\right)^{\mathrm{d} z}
\end{aligned}
\end{aligned}
$$




$$
\begin{aligned}
& \leqslant C \int_{Q_{i}^{k}} \frac{\left|b_{i}^{k}(z)\right|}{\left(\left|x-x_{i}^{k}\right|+2 r_{i}^{k}\right)^{n+\varepsilon}} \\
& \times\left(\sum_{j=2}^{\infty} \int_{2 r_{i}^{j} \leqslant\left|y-x_{i}^{k}\right|<2^{j+1} r_{i}^{k}}\left|\frac{|\Omega(y-z)|}{|y-z|^{n-\rho}}-\frac{\mid \Omega\left(y-x_{i}^{k}\right)}{\left|y-x_{i}^{k}\right|^{n-\rho}}\right|^{2}\right. \\
& \left.\times \frac{1}{\left|y-x_{i}^{k}\right|^{2 \rho-n-2 \varepsilon}} \mathrm{d} y\right)^{1 / 2} \mathrm{~d} z \\
& \leqslant C \int_{Q_{i}^{k}} \frac{\left|b_{i}^{k}(z)\right|}{\left(\left|x-x_{i}^{k}\right|+2 r_{i}^{k}\right)^{n+\varepsilon}} \\
& \times \sum_{j=2}^{\infty}\left(2^{j} r_{i}^{k}\right)^{\rho-n / 2-\varepsilon} \\
& \times\left(\int_{2^{j} r_{i}^{k} \leqslant\left|y-x_{i}^{k}\right|<2^{j+1} r_{i}^{k}}\left|\frac{|\Omega(y-z)|}{|y-z|^{n-\rho}}-\frac{\left|\Omega\left(y-x_{i}^{k}\right)\right|}{\left|y-x_{i}^{k}\right|^{n-\rho}}\right|^{2} \mathrm{~d} y\right)^{1 / 2} \mathrm{~d} z \\
& \leqslant C \int_{Q_{i}^{k}} \frac{\left|b_{i}^{k}(z)\right|}{\left(\left|x-x_{i}^{k}\right|+2 r_{i}^{k}\right)^{n+\varepsilon}} \sum_{j=2}^{\infty}\left(2^{j} r_{i}^{k}\right)^{\varepsilon} \\
& \times\left\{\frac{1}{2^{j}}+\frac{1}{2^{j \alpha}} \int_{\left|z-x_{i}^{k}\right| / 2^{j+1} r_{i}^{k}}^{\mid z-x_{i}^{k} / r^{j} r_{i}^{k}} \frac{w_{2}(\delta)}{\delta^{1+\alpha}} \mathrm{d} \delta\right\} \mathrm{d} z \\
& \leqslant C \int_{Q_{i}^{k}} \frac{\left|b_{i}^{k}(z)\right|\left(r_{i}^{k}\right)^{\varepsilon}}{\left(\left|x-x_{i}^{k}\right|+2 r_{i}^{k}\right)^{n+\varepsilon}} \mathrm{d} z \\
& \leqslant C 2^{k} \frac{\left(r_{i}^{k}\right)^{n+\varepsilon}}{\left|x-x_{i}^{k}\right|^{n+\varepsilon}} \text {. }
\end{aligned}
$$

From (61) to (66), we can obtain

$$
\begin{aligned}
& \sum_{k=k_{0}+1}^{\infty} \sum_{i} \int_{\left(2 A_{k_{0}}\right)^{c}} J_{4} w(x) \mathrm{d} x \\
& \leqslant C \sum_{k=k_{0}+1}^{\infty} \sum_{i} \int_{\left(2 A_{k_{0}}\right)^{c}}\left(J_{41}+J_{42}\right) w(x) \mathrm{d} x \\
& \leqslant C \sum_{k=k_{0}+1}^{\infty} \sum_{i} \int_{\left(2 A_{k_{0}}\right)^{c}}\left(J_{41}+J_{5}+J_{6}\right) w(x) \mathrm{d} x \\
& \leqslant C \sum_{k=k_{0}+1}^{\infty} \sum_{i} \int_{\left(2 A_{k_{0}}\right)^{c}}\left(J_{41}+J_{51}+J_{52}+J_{6}\right) w(x) \mathrm{d} x \\
& \leqslant C \sum_{k=k_{0}+1}^{\infty} \sum_{i} \int_{\left(2 A_{k_{0}}\right)^{c}} 2^{k} \\
& \quad \times\left(\frac{\left(r_{i}^{k}\right)^{n+\varepsilon / 2}}{\left|x-x_{i}^{k}\right|^{n+\varepsilon / 2}}+\frac{\left(r_{i}^{k}\right)^{n+\varepsilon}}{\left|x-x_{i}^{k}\right|^{n+\varepsilon}}\right) w(x) \mathrm{d} x
\end{aligned}
$$

$$
\begin{aligned}
& \leqslant C \sum_{k=k_{0}+1}^{\infty} 2^{k} \sum_{i}\left(r_{i}^{k}\right)^{\varepsilon / 2+n} \\
& \times \sum_{j=3}^{\infty} \int_{2^{j} \tau^{\left(k-k_{0}\right) / n} r_{i}^{k}<\left|x-x_{i}^{k}\right|<2^{j+1} \tau^{\left(k-k_{0}\right) / n} r_{i}^{k}} \frac{w(x)}{\left|x-x_{i}^{k}\right|^{n+\varepsilon / 2}} \mathrm{~d} x \\
& +C \sum_{k=k_{0}+1}^{\infty} 2^{k} \sum_{i}\left(r_{i}^{k}\right)^{\varepsilon+n} \\
& \times \sum_{j=3}^{\infty} \int_{2^{j} \tau^{\left(k-k_{0}\right) / n_{i_{i}^{k}}<\left|x-x_{i}^{k}\right|<2^{j+1}} \tau^{\left(k-k_{0}\right) / n} r_{i}^{k}} \frac{w(x)}{\left|x-x_{i}^{k}\right|^{n+\varepsilon}} \mathrm{d} x \\
& \leqslant C \sum_{k=k_{0}+1}^{\infty} 2^{k} \sum_{i}\left(r_{i}^{k}\right)^{\varepsilon / 2+n} \\
& \times \sum_{j=3}^{\infty} \frac{1}{\left(2^{j} r_{i}^{k}\right)^{\varepsilon / 2+n}} \int_{\left|x-x_{i}^{k}\right|<2^{j+1} \tau^{\left(k-k_{0}\right) / r_{r_{i}^{k}}^{k}}} w(x) \mathrm{d} x \\
& +\sum_{k=k_{0}+1}^{\infty} 2^{k} \sum_{i}\left(r_{i}^{k}\right)^{\varepsilon+n} \\
& \times \sum_{j=3}^{\infty} \frac{1}{\left(2^{j} r_{i}^{k}\right)^{\varepsilon+n}} \int_{\left|x-x_{i}^{k}\right|<2^{j+1} \tau^{\left(k-k_{0}\right) / n_{i}^{k}} r_{i}^{k}} w(x) \mathrm{d} x \\
& \leqslant C \sum_{k=k_{0}+1}^{\infty} 2^{k} \sum_{i} \sum_{j=3}^{\infty} 2^{-(j+1)(\varepsilon / 2)} \tau^{-\varepsilon\left(k-k_{0}\right) / 2 n} w\left(Q_{i}^{k}\right) \\
& +C \sum_{k=k_{0}+1}^{\infty} 2^{k} \sum_{i} \sum_{j=3}^{\infty} 2^{-(j+1) \varepsilon} \tau^{-\varepsilon\left(k-k_{0}\right) / n} w\left(Q_{i}^{k}\right) \\
& \leqslant C \sum_{k=k_{0}+1}^{\infty} \tau^{-\varepsilon\left(k-k_{0}\right) / 2 n}\|f\|_{W H_{w}^{1}} \\
& +C \sum_{k=k_{0}+1}^{\infty} \tau^{-\varepsilon\left(k-k_{0}\right) / n}\|f\|_{W H_{w}^{1}} \\
& \leqslant C\|f\|_{W H_{w}^{1}} \text {. }
\end{aligned}
$$

We conclude the proof of Theorem 3.

\section{Acknowledgments}

The authors would like to express their deep thanks to the referee for his/her very careful reading and many valuable comments and suggestions. Shuangping Tao is supported by National Natural Foundation of China (Grants nos. 11161042 and 11071250).

\section{References}

[1] E. M. Stein, "The development of square functions in the work of A. Zygmund," Bulletin of the American Mathematical Society, vol. 7, no. 2, pp. 359-376, 1982.

[2] C. E. Kenig, Harmonic Ayalysis Techniques for Second Order Elliptic Boundary Value Problems, vol. 83 of CBMS, American Mathematical Society, 1991. 
[3] S.-Y. A. Chang, J. M. Wilson, and T. H. Wolff, "Some weighted norm inequalities concerning the Schrödinger operators," Commentarii Mathematici Helvetici, vol. 60, no. 2, pp. 217-246, 1985.

[4] C. Fefferman and E. M. Stein, " $H^{p}$ spaces of several variables," Acta Mathematica, vol. 129, no. 3-4, pp. 137-193, 1972.

[5] C. Fefferman and E. M. Stein, "Some maximal inequalities," American Journal of Mathematics, vol. 93, pp. 107-115, 1971.

[6] E. M. Stein, "On the functions of Littlewood-Paley, Lusin, and Marcinkiewicz," Transactions of the American Mathematical Society, vol. 88, pp. 430-466, 1958.

[7] E. M. Stein, "On some funcions of Littlewood-Paley and Zygmund," Bulletin of the American Mathematical Society, vol. 67, pp. 99-101, 1961.

[8] E. M. Stein, Singular integrals and Differentiability Properties of Functions, Princeton University Press, Princeton, NJ, USA, 1970.

[9] L. Hörmander, "Estimates for translation invariant operators in $L^{p}$ spaces," Acta Mathematica, vol. 104, pp. 93-140, 1960.

[10] M. Sakamoto and K. Yabuta, "Boundedness of Marcinkiewicz functions," Studia Mathematica, vol. 135, no. 2, pp. 103-142, 1999.

[11] Y. Ding, S. Z. Lu, and K. Yabuta, "A problem on rough parametric Marcinkiewicz functions," Journal of the Australian Mathematical Society, vol. 72, no. 1, pp. 13-21, 2002.

[12] Y. Ding, S. Z. Lu, and Q. Y. Xue, "Parametrized area integrals on Hardy spaces and weak Hardy spaces," Acta Mathematica Sinica (English Series), vol. 23, no. 9, pp. 1537-1552, 2007.

[13] Y. Ding, S. Z. Lu, and Q. Y. Xue, "Parametrized Littlewood-Paley operators on Hardy and weak Hardy spaces," Mathematische Nachrichten, vol. 280, no. 4, pp. 351-363, 2007.

[14] H. B. Wang and Z. G. Liu, "Weighted estimates for parametrized Littlewood-Paley operators," Frontiers of Mathematics in China, vol. 6, no. 3, pp. 517-534, 2011.

[15] H. Wang, "Boundedness of intrinsic square functions on the weighted weak Hardy spaces," Integral Equations and Operator Theory, vol. 75, no. 1, pp. 135-149, 2013.

[16] C. Fefferman, N. M. Rivière, and Y. Sagher, "Interpolation between $H^{p}$ spaces: the real method," Transactions of the American Mathematical Society, vol. 191, pp. 75-81, 1974.

[17] R. Fefferman and F. Soria, “The space $H^{1}$," Studia Mathematica, vol. 85 , no. 1, pp. 1-16, 1987.

[18] H. P. Liu, "The weak $H^{p}$ spaces on homogeneous groups," in Harmonic analysis, Lecture Notes in Math., pp. 113-118, Springer, Berlin, Germany, 1991.

[19] T. S. Quek and D. C. Yang, "Calderón-Zygmund-type operators on weighted weak Hardy spaces over $\mathbb{R}^{n}$," Acta Mathematica Sinica (English Series), vol. 16, no. 1, pp. 141-160, 2000.

[20] B. Muckenhoupt, "Weighted norm inequalities for the Hardy maximal function," Transactions of the American Mathematical Society, vol. 165, pp. 207-226, 1972.

[21] J. García-Cuerva and J. L. Rubio de Francia, Weighted Norm Inequalities and Related Topics, North-Holland, Amsterdam, The Netherlands, 1985.

[22] Y. Ding and Q. Y. Xue, "Weighted $L^{p}$ boundedness for parametrized Littlewood-Paley operators," Taiwanese Journal of Mathematics, vol. 11, no. 4, pp. 1143-1165, 2007.

[23] Y. Ding and S. Z. Lu, "Homogeneous fractional integrals on Hardy spaces," The Tohoku Mathematical Journal, vol. 52, no. 1, pp. 153-162, 2000. 


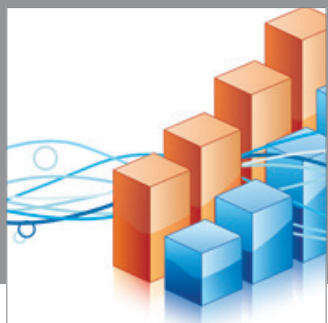

Advances in

Operations Research

mansans

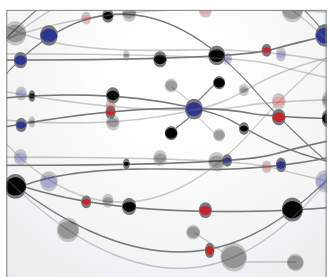

The Scientific World Journal
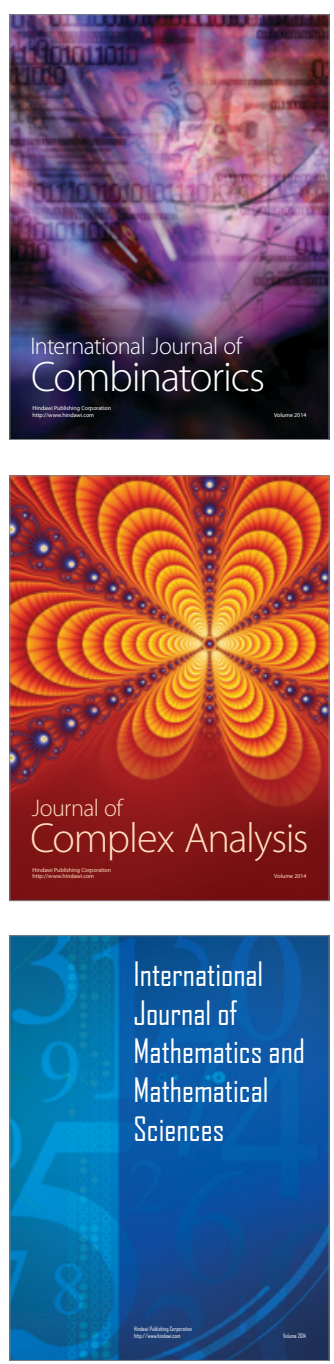
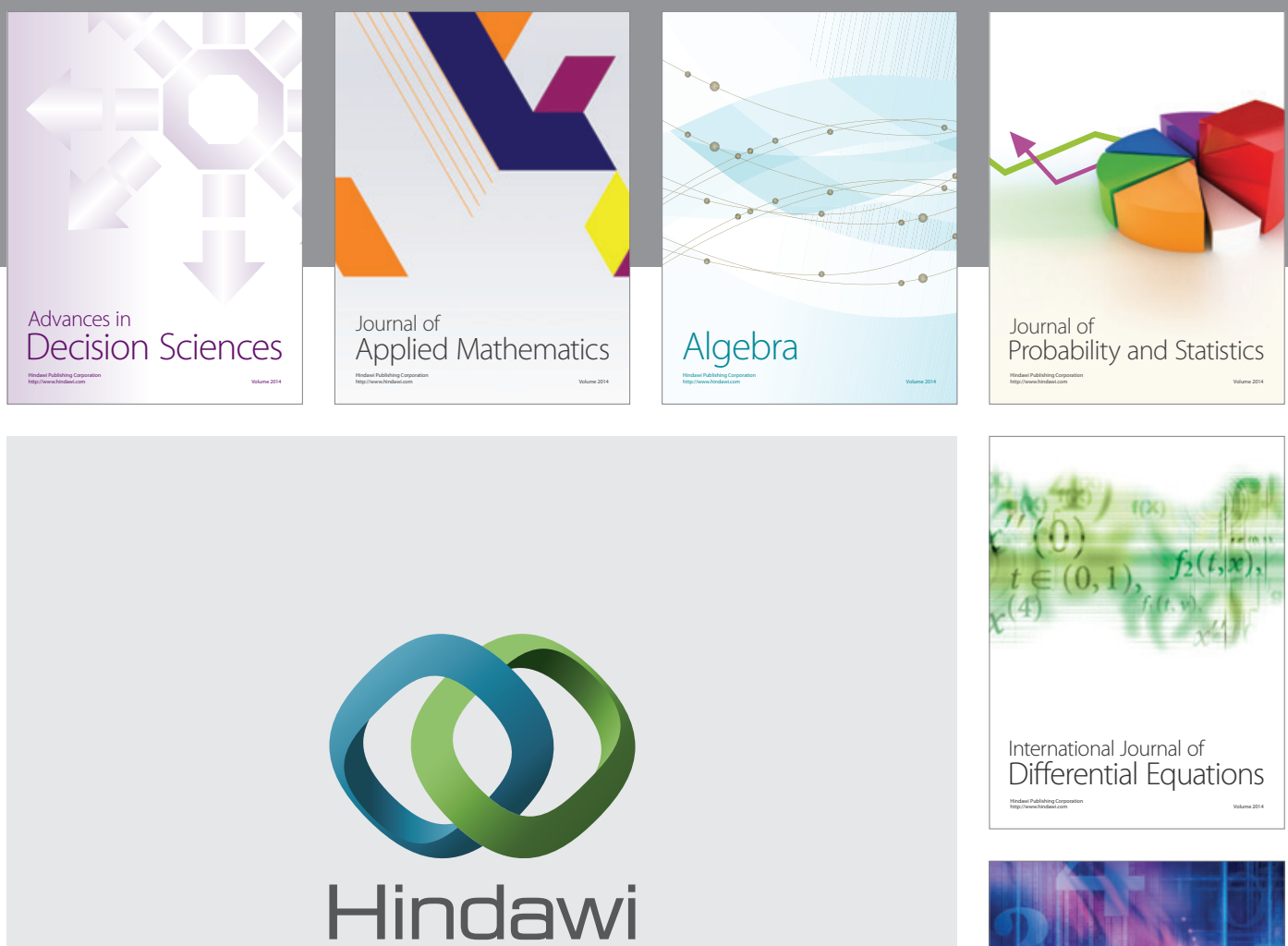

Submit your manuscripts at http://www.hindawi.com
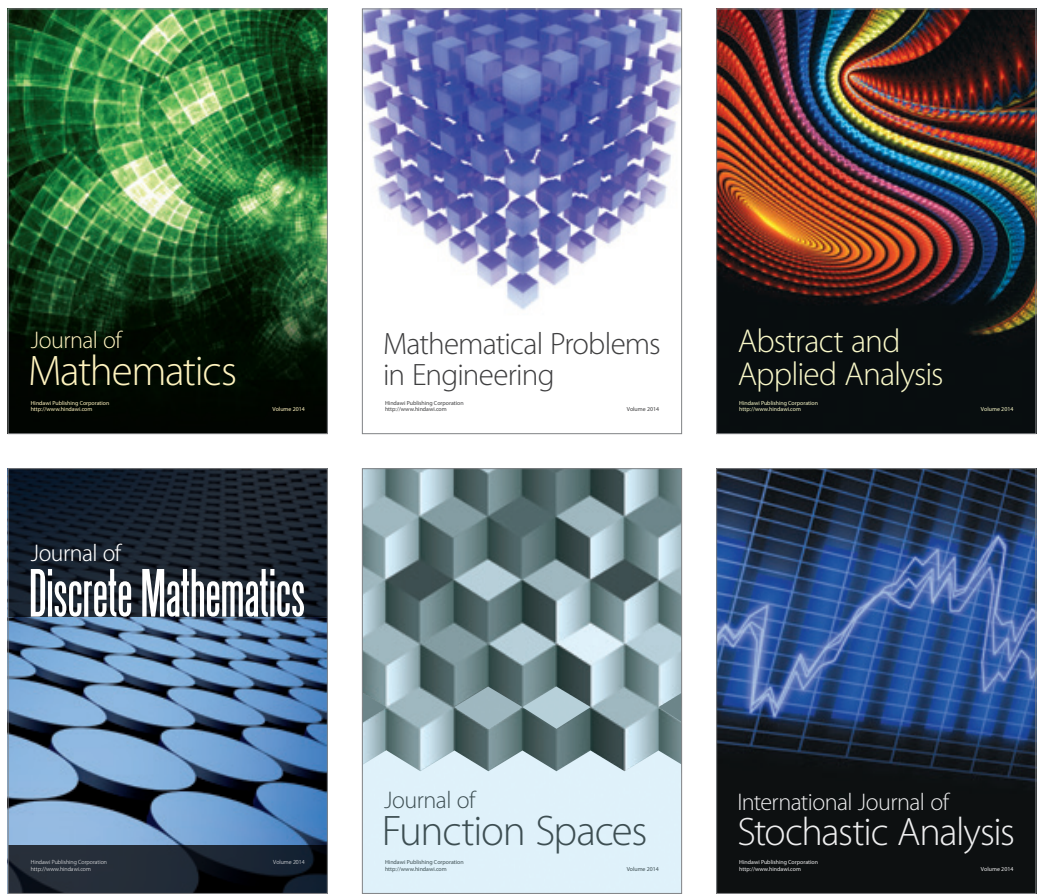

Journal of

Function Spaces

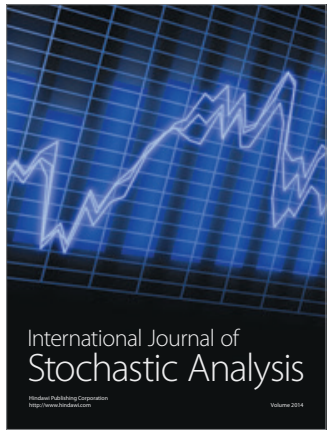

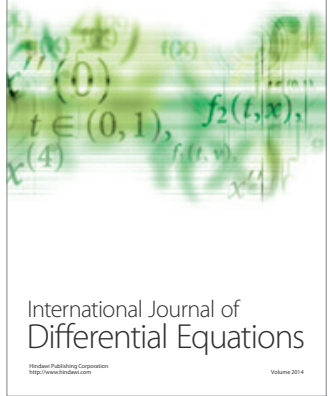
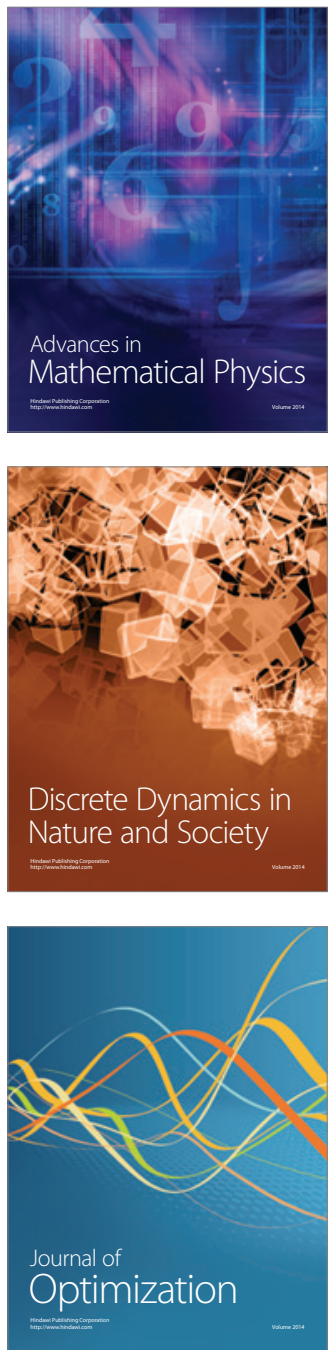